\title{
Planet formation around binary stars: Tatooine made easy
}

\author{
Benjamin C. Bromley \\ Department of Physics 86 Astronomy, University of Utah, \\ 115 S 1400 E, Rm 201, Salt Lake City, UT 84112 \\ bromley@physics.utah.edu \\ Scott J. Kenyon \\ Smithsonian Astrophysical Observatory, \\ 60 Garden St., Cambridge, MA 02138 \\ skenyon@cfa.harvard.edu
}

\begin{abstract}
We examine characteristics of circumbinary orbits in the context of current planet formation scenarios. Analytical perturbation theory predicts the existence of nested circumbinary orbits that are generalizations of circular paths around a single star. These orbits have forced eccentric motion aligned with the binary as well as higher frequency oscillations, yet they do not cross, even in the presence of massive disks and perturbations from large planets. For this reason, dissipative gas and planetesimals can settle onto these "most circular" orbits, facilitating the growth of protoplanets. Outside a region close to the binary where orbits are generally unstable, circumbinary planets form in much the same way as their cousins around a single star. Here, we review the theory and confirm its predictions with a suite of representative simulations. We then consider the circumbinary planets discovered with NASA's Kepler satellite. These Neptune- and Jupiter-size planets, or their planetesimal precursors, may have migrated inward to reach their observed orbits, since their current positions are outside of unstable zones caused by overlapping resonances. In situ formation without migration seems less likely, only because the surface density of the protoplanetary disks must be implausibly high. Otherwise, the circumbinary environment is friendly to planet formation, and we expect that many Earth-like "Tatooines" will join the growing census of circumbinary planets.
\end{abstract}

Subject headings: planetary systems - planets and satellites: formation - planets and satellites: dynamical evolution and stability - planet disk interactions binaries: close - stars: individual (Kepler-16) 


\section{Introduction}

Planet formation is robust. Transit detections by the Kepler satellite (e.g., Borucki et al. 2011; Howard et al. 2012), radial velocity campaigns (Cumming et al. 2008; Howard et al. 2010; Zechmeister et al. 2013; Mayor et al. 2014), direct imaging surveys (Macintosh et al. 2014; Tamura 2014), and gravitational lensing studies (Gould et al. 2010) suggest that many if not all stars host planets (Youdin 2011; Dong \& Zhu 2013). To date, there are over 1500 confirmed planets, and several thousand candidates (Han et al. 2014).

The vast majority of the known planets orbit a single star (e.g., Mavor et al. 2011; Cassan et al. 2012; Burke et al. 2014; Mullally et al. 2015). Their formation in this setting is straightforward to describe, even if certain key details are not well understood (e.g., Safronov 1969; Wetherill 1980; Goldreich et al. 2004; Youdin \& Kenvon 2013). The overall process takes many small solid particles of dust and concentrates this mass into a few large objects. Coagulation - growth through sticking or merging of planetesimals - is driven by collisions. Low relative velocities favor growth, but can also slow it down. Higher velocities can speed up the growth rate, but can also lead to destructive collisions. Scattering and gravitational interactions pump up relative velocities, while collisional damping and dynamical friction slow things down. A balance between these processes enables planets to emerge from the dust.

In addition to the dozens of planets known to orbit one member of a stellar binary (e.g., $\alpha$ Cen B; see Schneider et al. 2011), a handful of planets are known to orbit both binary partners. Kepler-16b - the first circumbinary planet discovered by the Kepler mission - is a Saturn-mass planet at an orbital distance of about 0.7 AU (Doyle et al. 2011). Its binary host consists of a $0.7 M_{\odot}$ K-type star and a $0.2 M_{\odot}$ red dwarf at an orbital separation of $0.22 \mathrm{AU}$. Since then, six more "Tatooines" have been reported in the Kepler data set (see Table 1, below). They are typically Neptune- or Jupiter-size, and all orbit their hosts at distances within roughly 1 AU. One binary, Kepler 47, hosts two such planets (Orosz et al. 2012a). A few more massive circumbinary planets are known or suspected, but these objects orbit at much greater distances compared with the binary semimajor axis (e.g., Beuermann et al. 2011).

The circumbinary planets that orbit close to their hosts provide unique challenges for planet formation theory. The central stars strongly perturb the region around them, clearing out orbits to distances of 2-5 times the binary separation (Holman \& Wiegert 1999). Similarly, circumstellar disks get eroded from the outside by the binary partner. In either case, secular excitations from the binary potential drive orbital crossings and destructive collisions (Moriwaki \& Nakagawa 2004; Meschiari 2012; Paardekooper et al. 2012; Rafikov 2013; Lines et al. 2014). Thus, planets may not be able to grow near their binary host. 
To resolve this problem, planets may form at more remote distances, where the timevarying part of the binary potential is weak. To arrive at their observed locations, they must then migrate through the circumbinary gaseous disk or scatter with neighboring gas giants (Pierens \& Nelson 2008a, b) . While the simulations of this process are compelling (e.g., Kley \& Haghighipour 2014), there are uncertainties about starting conditions, typically a set of planetary cores placed into a steady state disk. It is unclear if the simultaneous growth of planetesimals and dissipation of the disk can conspire to produce cores poised to migrate into their observed orbital positions.

Toward understanding how circumbinary planets form, we re-examine a fundamental issue: the nature of planetesimal orbits around binary stars. Following the approach of Lee \& Peale (2006) and Leung \& Lee (2013), we describe a family of nested, stable circumbinary orbits that have minimal radial excursions and never intersect. While they are not exactly circular, these orbits play the same role as circular paths around a single star. Gas and particles can damp to these orbits as they dynamically cool, avoiding the destructive secular excitations reported in previous work. Thus planetesimals may grow in situ to full-fledged planets.

We organize this paper to provide an introduction to the Lee-Peale-Leung analytical theory of circumbinary orbits $(\S 2)$, followed by numerical examples ( 33$)$. We then discuss the role these orbits play in planet formation $(\S 4)$, and close with a comparison to observations $(\S 5)$, along with a summary and predictions of the ideas presented here $(\S 6)$.

\section{The circumbinary environment}

Planet formation relies on the ability of solid particles — dust, planetesimals, protoplanets - to interact gently. Around a single star, the family of nested (concentric) circular orbits offers this possibility. Particles on coplanar circular orbits coexist without any collisions. Gravitational interactions among particles (and with coexisting gas) induce random motions about these circular orbits, which enables particles to merge into larger objects. Unchecked, gravitational interactions grow indefinitely and lead to destructive collisions among particles (e.g., Goldreich \& Tremaine 1978). Dynamical cooling (through collisional damping, dynamical friction, or gas drag) is essential (e.g., Goldreich et al. 2004; Youdin \& Kenyon 2013). When orbiting particles cool, they need some common set of trajectories on which to settle. The family of circular orbits provides this non-intersecting, collisionless haven for particles in cold circumstellar disks.

A central binary dramatically alters these orbital dynamics (e.g., Holman \& Wiegert 
1999; Musielak et al. 2005; Pichardo et al. 2005; Doolin \& Blundell 2011). For a primary and secondary with comparable masses and binary eccentricity, $e_{\text {bin }}$, satellite orbits are unstable inside of a critical radius, $a_{\text {crit }}$, which is at least twice the binary separation, $a_{\text {bin }}$. Holman \& Wiegert (1999) derive an approximation for $a_{\text {crit }}$ from direct simulation of circumbinary particles:

$$
\begin{aligned}
a_{\text {crit }} \approx & 1.60+5.10 e_{\text {bin }}-2.22 e_{\mathrm{bin}}^{2}+4.12 \frac{M_{s}}{M_{p}+M_{s}}-4.27 e_{\mathrm{bin}} \frac{M_{s}}{M_{p}+M_{s}} \\
& -5.09 \frac{M_{s}^{2}}{\left(M_{p}+M_{s}\right)^{2}}+4.61 e_{\mathrm{bin}}^{2} \frac{M_{s}^{2}}{\left(M_{p}+M_{s}\right)^{2}},
\end{aligned}
$$

where $M_{p}$ and $M_{s}$ are the masses of the primary and secondary, respectively (see also Pichardo et al. 2005, 2008). Inside this orbital distance, particles are cleared, creating a cavity around the binary.

Particles beyond the critical distance can be on stable, non-Keplerian orbits. In addition to their response to the central mass of the binary, these satellites also experience forced motion, driven by the binary's time-varying potential. This perturbation prevents particles from maintaining circular or eccentric orbits. Instead, particles may achieve a "most circular orbit," defined as having the smallest radial excursion about some guiding center, orbiting at some constant radius $R_{\mathrm{g}}$ and angular speed $\Omega_{\mathrm{g}}$ in the plane of the binary (Lee \& Peale 2006; Youdin et al. 2012). More generally, eccentric circumbinary orbits may be composed of epicyclic motion about $R_{\mathrm{g}}$, as in the Keplerian case, superimposed on the most circular orbit.

In the rest of this section, we investigate existing analytic theory for circumbinary orbits and applications for planet formation. We also include a brief discussion of instabilities and resonances, as a prelude to numerical simulations in $\S 3$.

\subsection{Analytical theory of circumbinary orbits}

To describe satellite orbits about a central binary, we follow the analytic theories of Lee \& Peale (2006) and Leung \& Lee (2013), based on the restricted three-body problem (see Szebehely 1967; Murray \& Dermott 1999). In this framework, a satellite's position and momentum come from equations of motion in the potential of a stellar binary. This strategy differs from previous work based on secular perturbation theory (e.g., Heppenheimer 1978; Marzari \& Scholl 2000; Moriwaki \& Nakagawa 2004; Rafikov 2013). In that approach, a satellite's osculating orbital elements, defined with respect to a central point mass, evolve according to an orbit-averaged disturbing function (e.g., Murray \& Dermott 1999). However, these elements do not accurately track the orbits of the Kepler circumbinary planets; 
the binary induces significant motion on dynamical time scales. Although modifications to the secular theory can accommodate this extra motion (Georgakarakos \& Eggl 2015), any additional non-gravitational processes like aerodynamic drag or planetesimal collisions are more easily described in terms of positions and momenta. For these reasons we adopt the approach of Lee \& Peale (2006) and Leung \& Lee (2013).

The starting point of the Lee-Peale-Leung analysis is the gravitational potential of the binary:

$$
\Phi=-\frac{G M_{p}}{\sqrt{R^{2}+z^{2}+R_{p}^{2}+2 R R_{p} \cos \Delta \phi}}-\frac{G M_{s}}{\sqrt{R^{2}+z^{2}+R_{s}^{2}-2 R R_{s} \cos \Delta \phi}},
$$

where $G$ is the gravitational constant and $\Delta \phi$ is angle between the secondary and the satellite in a reference frame with the binary's center of mass at the origin. In this frame, the massless satellite is at radial position $R$ in the plane of the binary and has altitude $z$ above this plane. The terms $R_{p}$ and $R_{s}$ denote the orbital distances of the primary and secondary.

To make headway, this potential is expanded in terms of the angle cosines, converted from powers $\left(\cos ^{k}(\Delta \phi)\right)$ to multiple-angle form $(\cos (k \Delta \phi))$. For eccentric binaries, Leung \& Lee (2013) also expand the potential to first order in the binary eccentricity, $e_{\text {bin }}$, using the epicyclic approximation to describe the variation in binary separation and phase. Then they seek solutions for the excursion of the satellite from a guiding center on a circular orbit of radius $R_{\mathrm{g}}$. The excursions in radial, azimuthal and altitude coordinates are $\delta R, \delta \phi$, and $\delta z$ (which is identically $z$, since all vertical motions are excursions from the guiding center orbiting in plane of the binary). Their solution can be estimated by writing the equations of motion and keeping only terms linear in the perturbation coordinates and in the binary eccentricity. Between the expansion of the potential and this linearization, the problem reduces to the form of a simple, driven harmonic oscillator.

To follow this prescription, we focus on motion in the binary's orbital plane. The potential is

$$
\begin{aligned}
\Phi \approx \sum_{k=0}^{\infty}\left\{\Phi_{0 k} \cos (k \Delta \phi)\right. & +e_{\text {bin }}\left[\Phi_{0 k}^{e} \cos (k \Delta \phi) \cos \left(\Omega_{\mathrm{bin}} t\right)\right. \\
+ & \left.\left.2 k \Phi_{0 k} \sin (k \Delta \phi) \sin \left(\Omega_{\mathrm{bin}} t\right)\right]\right\} \quad[z=0]
\end{aligned}
$$

where $\Omega_{\mathrm{bin}}$ is the mean motion of the binary $\left(\Omega_{\mathrm{bin}}{ }^{2}=G\left(M_{p}+M_{s}\right) / a_{\mathrm{bin}}{ }^{3}\right)$, and our choice of time $t$ fixes the orbital phases of the binary and satellite. The potentials $\Phi_{0 k}$ are Fourier coefficients derived from the expansion of the potential in terms of $\cos (\Delta \phi)$ evaluated at $e_{\text {bin }}=0$, while the $\Phi_{0 k}^{e}$ are those same coefficients giving the first-order terms of a Taylor 
series in $e_{\text {bin. }}$. Examples of these coefficients are

$$
\begin{aligned}
& \Phi_{00}=-\frac{G M}{R_{\mathrm{g}}}-\frac{G \mu}{R_{\mathrm{g}}}\left[\frac{1}{4} \frac{a_{\mathrm{bin}}{ }^{2}}{R_{\mathrm{g}}{ }^{2}}+\frac{9}{64} \frac{\left(M_{p}^{2}+M_{s}^{2}\right)}{M^{2}} \frac{a_{\mathrm{bin}}{ }^{4}}{R_{\mathrm{g}}{ }^{4}}+\frac{25}{256} \frac{\left(M_{p}^{4}+M_{s}^{4}\right)}{M^{4}} \frac{a_{\mathrm{bin}}{ }^{6}}{R_{\mathrm{g}}{ }^{6}}+\ldots\right] \\
& \Phi_{01}=-\frac{G \mu}{R_{\mathrm{g}}}\left[\frac{3}{8} \frac{\left(M_{p}-M_{s}\right)}{M} \frac{a_{\mathrm{bin}}{ }^{3}}{R_{\mathrm{g}}{ }^{3}}+\frac{15}{64} \frac{\left(M_{p}^{4}-M_{s}^{4}\right)}{M^{4}} \frac{a_{\mathrm{bin}}{ }^{5}}{R_{\mathrm{g}}{ }^{5}}+\ldots\right] \\
& \Phi_{02}=-\frac{G \mu}{R_{\mathrm{g}}}\left[\frac{3}{4} \frac{a_{\mathrm{bin}}{ }^{2}}{R_{\mathrm{g}}{ }^{2}}+\frac{5}{16} \frac{\left(M_{p}^{3}+M_{s}^{3}\right)}{M^{3}} \frac{a_{\mathrm{bin}}}{R_{\mathrm{g}}{ }^{4}}+\frac{105}{512} \frac{\left(M_{p}^{5}+M_{s}^{5}\right)}{M^{5}} \frac{a_{\mathrm{bin}}{ }^{6}}{R_{\mathrm{g}}{ }^{6}}+\ldots\right] \\
& \Phi_{03}=-\frac{G \mu}{R_{\mathrm{g}}}\left[\frac{5}{8} \frac{\left(M_{p}-M_{s}\right)}{M} \frac{a_{\mathrm{bin}}{ }^{3}}{R_{\mathrm{g}}{ }^{3}}+\frac{35}{128} \frac{\left(M_{p}^{4}-M_{s}^{4}\right)}{M^{4}} \frac{a_{\mathrm{bin}}{ }^{5}}{R_{\mathrm{g}}{ }^{5}}+\ldots\right] \\
& \Phi_{04}=-\frac{G \mu}{R_{\mathrm{g}}}\left[\frac{35}{64} \frac{\left(M_{p}^{3}+M_{s}^{3}\right)}{M^{3}} \frac{a_{\mathrm{bin}}{ }^{4}}{R_{\mathrm{g}}{ }^{4}}+\frac{63}{256} \frac{\left(M_{p}^{5}+M_{s}^{5}\right)}{M^{5}} \frac{a_{\mathrm{bin}}{ }^{6}}{R_{\mathrm{g}}{ }^{6}}+\ldots\right] \\
& \Phi_{05}=-\frac{G \mu}{R_{\mathrm{g}}}\left[\frac{63}{128} \frac{\left(M_{p}^{4}-M_{s}^{4}\right)}{M^{4}} \frac{a_{\mathrm{bin}}{ }^{5}}{R_{\mathrm{g}}{ }^{5}}+\ldots\right] ;
\end{aligned}
$$

and

$$
\begin{aligned}
\Phi_{00}^{e} & =-\frac{G \mu}{R_{\mathrm{g}}}\left[\frac{1}{2} \frac{a_{\mathrm{bin}}{ }^{2}}{R_{\mathrm{g}}{ }^{2}}+\frac{9}{16} \frac{\left(M_{p}^{3}+M_{s}^{3}\right)}{M^{3}} \frac{a_{\mathrm{bin}}{ }^{4}}{R_{\mathrm{g}}{ }^{4}}+\frac{75}{128} \frac{\left(M_{p}^{5}+M_{s}^{5}\right)}{M^{5}} \frac{a_{\mathrm{bin}}{ }^{6}}{R_{\mathrm{g}}{ }^{6}}+\ldots\right] \\
\Phi_{01}^{e} & =-\frac{G \mu}{R_{\mathrm{g}}}\left[\frac{9}{8} \frac{\left(M_{p}-M_{s}\right)}{M} \frac{a_{\mathrm{bin}}{ }^{3}}{R_{\mathrm{g}}{ }^{3}}+\frac{75}{64} \frac{\left(M_{p}^{4}-M_{s}^{4}\right)}{M^{4}} \frac{a_{\mathrm{bin}}{ }^{5}}{R_{\mathrm{g}}{ }^{5}}+\ldots\right] \\
\Phi_{02}^{e} & =-\frac{G \mu}{R_{\mathrm{g}}}\left[\frac{3}{2} \frac{a_{\mathrm{bin}}{ }^{2}}{R_{\mathrm{g}}{ }^{2}}+\frac{5}{4} \frac{\left(M_{p}^{3}+M_{s}^{3}\right)}{M^{3}} \frac{a_{\mathrm{bin}}{ }^{4}}{R_{\mathrm{g}}{ }^{4}}+\frac{315}{256} \frac{\left(M_{p}^{5}+M_{s}^{5}\right)}{M^{5}} \frac{a_{\mathrm{bin}}{ }^{6}}{R_{\mathrm{g}}{ }^{6}}+\ldots\right] \\
\Phi_{03}^{e} & =-\frac{G \mu}{R_{\mathrm{g}}}\left[\frac{15}{8} \frac{\left(M_{p}-M_{s}\right)}{M} \frac{a_{\mathrm{bin}}{ }^{3}}{R_{\mathrm{g}}{ }^{3}}+\frac{175}{128} \frac{\left(M_{p}^{4}-M_{s}^{4}\right)}{M^{4}} \frac{a_{\mathrm{bin}}{ }^{5}}{R_{\mathrm{g}}{ }^{5}}+\ldots\right] \\
\Phi_{04}^{e} & =-\frac{G \mu}{R_{\mathrm{g}}}\left[\frac{35}{16} \frac{\left(M_{p}^{3}+M_{s}^{3}\right)}{M^{3}} \frac{a_{\mathrm{bin}}{ }^{4}}{R_{\mathrm{g}}{ }^{4}}+\frac{189}{128} \frac{\left(M_{p}^{5}+M_{s}^{5}\right)}{M^{5}} \frac{a_{\mathrm{bin}}{ }^{6}}{R_{\mathrm{g}}{ }^{6}}+\ldots\right] \\
\Phi_{05}^{e} & =-\frac{G \mu}{R_{\mathrm{g}}}\left[\frac{315}{256} \frac{\left(M_{p}^{4}-M_{s}^{4}\right)}{M^{4}} \frac{a_{\mathrm{bin}}{ }^{5}}{R_{\mathrm{g}}{ }^{5}}+\ldots\right] .
\end{aligned}
$$

where $M=M_{p}+M_{s}$ is the total mass and $\mu=M_{p} M_{s} /\left(M_{p}+M_{s}\right)$ is the reduced mass 1 . The subscripts $j k$ designate that each term is measured in the plane of the binary $(j=0)$, and is the $k^{\text {th }}$ harmonic as in Equation (4). The missing terms are of order $\left(a_{\text {bin }} / R_{\mathrm{g}}\right)^{7}$, which can be as large as a percent in an idealized system, and a fraction of a percent in observed binaries.

\footnotetext{
${ }^{1}$ In some previous studies, " $\mu$ " is defined as the ratio of the secondary's mass to the total mass. Thus, our $\mu / M$ is equal to " $\mu(1-\mu)$ " in Holman \& Wiegert (1999), for example.
} 
From the time-averaged potential $\left(\Phi_{00}\right.$ in Equation (5) ), we obtain the angular speed of the guiding center, $\Omega_{\mathrm{g}}$. It follows from

$$
\left.\Omega_{\mathrm{g}}{ }^{2} \equiv \frac{1}{R_{\mathrm{g}}} \frac{d \Phi_{00}}{d R}\right|_{R_{\mathrm{g}}}=\frac{G M}{R_{\mathrm{g}}{ }^{3}}\left\{1+\frac{\mu}{M}\left[\frac{3}{4} \frac{a_{\mathrm{bin}}{ }^{2}}{R_{\mathrm{g}}{ }^{2}}+\frac{45}{64} \frac{\left(M_{p}^{3}+M_{s}^{3}\right)}{M^{3}} \frac{a_{\mathrm{bin}}{ }^{4}}{R_{\mathrm{g}}{ }^{4}}+\ldots\right]\right\} .
$$

The square root of $\Omega_{\mathrm{g}}{ }^{2}$ is the mean motion of the satellite. The satellite's epicyclic and vertical frequencies are

$$
\begin{aligned}
& \left.\kappa_{\mathrm{e}}^{2} \equiv R_{\mathrm{g}} \frac{d \Omega_{\mathrm{g}}{ }^{2}}{d R}\right|_{R_{\mathrm{g}}}+4 \Omega_{\mathrm{g}}{ }^{2}=\frac{G M}{R_{\mathrm{g}}{ }^{3}}\left\{1-\frac{\mu}{M}\left[\frac{3}{4} \frac{a_{\mathrm{bin}}{ }^{2}}{R_{\mathrm{g}}{ }^{2}}+\frac{135}{64} \frac{\left(M_{p}^{3}+M_{s}^{3}\right)}{M^{3}} \frac{a_{\mathrm{bin}}{ }^{4}}{R_{\mathrm{g}}{ }^{4}}+\ldots\right]\right\} \\
& \left.\nu_{\mathrm{i}}^{2} \equiv \frac{1}{z} \frac{d \Phi}{d z}\right|_{z=0, R_{\mathrm{g}}}=\frac{G M}{R_{\mathrm{g}}{ }^{3}}\left\{1+\frac{\mu}{M}\left[\frac{9}{4} \frac{a_{\mathrm{bin}}{ }^{2}}{R_{\mathrm{g}}{ }^{2}}+\frac{225}{64} \frac{\left(M_{p}^{3}+M_{s}^{3}\right)}{M^{3}} \frac{a_{\mathrm{bin}}{ }^{4}}{R_{\mathrm{g}}{ }^{4}}+\ldots\right]\right\}
\end{aligned}
$$

corresponding to the eccentricity and any motion out of the plane of the binary (for details regarding the motion out of the orbital plane, see Lee \& Peale 2006). In the limit that the binary separation goes to zero, or when the binary mass ratio is extreme, both $\kappa_{\mathrm{e}}$ and $\nu_{\mathrm{i}}$ become the Keplerian mean motion.

Forced oscillations experienced by a satellite depend on the synodic frequency,

$$
\omega_{\text {syn }}=\Omega_{\text {bin }}-\Omega_{\mathrm{g}} .
$$

In general, $\omega_{\text {syn }}$ is just the average angular speed of the satellite in a reference frame that rotates with the mean motion of the binary. For a circular binary $\left(e_{\text {bin }}=0\right)$, the time varying force felt by the satellite depends only on this frequency and its harmonics. In the case of an eccentric binary, the orbital frequency of the binary also enters into the potential.

\subsubsection{Equations of motion}

Derivatives of the potential yield equations of motion in the excursion coordinates $(\delta R, \delta \phi, z)$. The strategy of Lee \& Peale $(2006)$ is to cast these equations in the form of a forced harmonic oscillator with natural frequencies $\kappa_{\mathrm{e}}$ (for $\delta R$ and $\delta \phi$ ), $\nu_{\mathrm{i}}$ (for the $z$ coordinate) and driving frequencies involving $\Omega_{\text {bin }}$ and $\omega_{\text {syn }}$. The solutions in terms of the full cylindrical coordinates are (Equations (27), (31) and (35) in Leung \& Lee 2013):

$$
\begin{aligned}
& R(t)=R_{\mathrm{g}}\left\{1-e_{\text {free }} \cos \left(\kappa_{\mathrm{e}} t+\psi_{e}\right)-\sum_{k=1}^{\infty} C_{k} \cos \left(k \omega_{\mathrm{syn}} t\right)\right. \\
& \left.-e_{\mathrm{bin}}\left[\tilde{C}_{0}^{e} \cos \left(\Omega_{\mathrm{bin}} t\right)+\sum_{k=1}^{\infty} \tilde{C}_{k}^{+} \cos \left(k \omega_{\mathrm{syn}} t+\Omega_{\mathrm{bin}} t\right)+\tilde{C}_{k}^{-} \cos \left(k \omega_{\mathrm{syn}} t-\Omega_{\mathrm{bin}} t\right)\right]\right\}
\end{aligned}
$$




$$
\begin{aligned}
& \phi(t)=\Omega_{\mathrm{g}}\left\{t+\frac{2 e_{\mathrm{free}}}{\kappa_{\mathrm{e}}} \sin \left(\kappa_{\mathrm{e}} t+\psi_{e}\right)+\sum_{k=1}^{\infty} \frac{D_{k}}{k \omega_{\mathrm{syn}}} \sin \left(k \omega_{\mathrm{syn}} t\right)\right. \\
& \left.+e_{\mathrm{bin}}\left[\frac{\tilde{D}_{0}^{e}}{\Omega_{\mathrm{bin}}} \cos \left(\Omega_{\mathrm{bin}} t\right)+\sum_{k=1}^{\infty} \frac{\tilde{D}_{k}^{+} \sin \left(k \omega_{\mathrm{syn}} t+\Omega_{\mathrm{bin}} t\right)}{k \omega_{\mathrm{syn}}+\Omega_{\mathrm{bin}}}+\frac{\tilde{D}_{k}^{-} \sin \left(k \omega_{\mathrm{syn}} t-\Omega_{\mathrm{bin}} t\right)}{k \omega_{\mathrm{syn}}-\Omega_{\mathrm{bin}}}\right]\right\} \\
& z(t)=i R_{\mathrm{g}} \cos \left(\nu_{\mathrm{i}} t+\psi_{i}\right),
\end{aligned}
$$

where $e_{\text {free }}$ is the "free" eccentricity, $i$ is the inclination, and the phase angles $\psi_{e}$ and $\psi_{i}$ are constants. The coefficients are

$$
\begin{aligned}
C_{k} & =\frac{1}{R_{\mathrm{g}}\left(\kappa_{\mathrm{e}}^{2}-k^{2} \omega_{\mathrm{syn}}^{2}\right)}\left[\frac{d \Phi_{0 k}}{d R}-\frac{2 \Omega_{\mathrm{g}} \Phi_{0 k}}{R_{\mathrm{g}} \omega_{\mathrm{syn}}}\right]_{R_{\mathrm{g}}} \\
\tilde{C}_{0}^{e} & =-\frac{1}{R_{\mathrm{g}}\left(\kappa_{\mathrm{e}}^{2}-\Omega_{\mathrm{bin}}{ }^{2}\right)}\left[\frac{d \Phi_{00}^{e}}{d R}\right]_{R_{\mathrm{g}}} \\
\tilde{C}_{k}^{ \pm} & =\frac{1}{R_{\mathrm{g}}\left[\kappa_{\mathrm{e}}^{2}-\left(k \omega_{\mathrm{syn}} \pm \Omega_{\mathrm{bin}}\right)^{2}\right]}\left[k \frac{d \Phi_{0 k}}{d R}-\frac{1}{2} \frac{d \Phi_{0 k}^{e}}{d R}-\frac{k \Omega_{\mathrm{g}}\left( \pm 2 k \Phi_{0 k}-\Phi_{0 k}^{e}\right)}{R\left(k \omega_{\mathrm{syn}} \pm \Omega_{\mathrm{bin}}\right)}\right]_{R_{\mathrm{g}}} \\
D_{k} & =2 C_{k}+\left[\frac{\Phi_{0 k}}{R_{\mathrm{g}}{ }^{2} \Omega_{\mathrm{g}} \omega_{\mathrm{syn}}}\right]_{R_{\mathrm{g}}} \\
\tilde{D}_{0}^{e} & =2 \tilde{C}_{0}^{e} \\
\tilde{D}_{k}^{ \pm} & =2 \tilde{C}_{k}^{ \pm}+\left[\frac{k\left( \pm 2 k \Phi_{0 k}-\Phi_{0 k}^{e}\right)}{2 R_{\mathrm{g}}{ }^{2} \Omega_{\mathrm{g}}\left(k \omega_{\mathrm{syn}} \pm \Omega_{\mathrm{bin}}\right)}\right]_{R_{\mathrm{g}}}
\end{aligned}
$$

(Equations (28-30) and (32-34) in Leung \& Lee 2013) 2

\subsubsection{Components of the orbital motion}

From the Leung \& Lee (2013) solutions, we see that circumbinary orbits may be broken up into independent modes. The first mode is forced motion, prescribed by the characteristics of the binary and the orbital distance of the satellite from the center of mass. The second mode is "free" motion, fully analogous to eccentricity and inclination of orbits around a single central mass, except that the epicyclic excursions are relative to an orbiting reference frame locked into forced motion, as opposed to a circular guiding center.

\footnotetext{
${ }^{2}$ The coefficients $\tilde{C}$ and $\tilde{D}$ are equal to the corresponding $C$ and $D$ in Leung \& Lee (2013) divided by the binary eccentricity. For example, $\tilde{C}_{k}^{+}=C_{k}^{+} / e_{\text {bin }}$ and $\tilde{C}_{0}^{e}=C_{0} / e_{\text {bin }}$. Our choice allows the dependence on binary eccentricity to appear explicitly in the solutions of the excursion variables.
} 
The forced motion itself can be broken down into parts, including fast, driven oscillations at the synodic frequency and the binary's orbital frequency, plus the slower epicyclic oscillations operating at the orbital frequency, $\Omega_{\mathrm{g}}$. The higher frequency contributions have radial excursion amplitudes that scale as

$$
\left\{C_{2}, \tilde{C}_{2}^{ \pm}\right\} \times R_{\mathrm{g}} \sim \frac{\mu}{M}\left(\frac{a_{\mathrm{bin}}}{R_{\mathrm{g}}}\right)^{5} R_{\mathrm{g}}
$$

to leading order in $a_{\text {bin }} / R_{\mathrm{g}}$ (e.g., Leung \& Lee 2013). The slower epicyclic motion is associated with the forced eccentric orbit (Heppenheimer 1978). Its contribution to the radial excursions is typically much larger, scaling as

$$
e_{\text {force }} R_{\mathrm{g}}=\tilde{C}_{1}^{-} R_{\mathrm{g}} \approx \frac{5}{4} \frac{M_{p}-M_{s}}{M} e_{\mathrm{bin}} a_{\mathrm{bin}}
$$

When the binary eccentricity is large, we rewrite the solutions to highlight the role of the forced eccentricity. For example, the radial coordinate becomes

$$
R(t)=R_{\mathrm{g}}\left[1-e_{\text {free }} \cos \left(\kappa_{\mathrm{e}} t+\psi_{e}\right)-e_{\text {force }} \cos \left(\Omega_{\mathrm{g}} t\right)+\ldots .\right]
$$

This form of the solution is familiar from secular perturbation theory Murray \& Dermott 1999).

An important feature of the forced eccentric orbit is that it does not precess. Its argument of periastron is locked in line with the argument of periastron for the binary. All other components of the forced motion are also synchronized to the binary's orbital frequency, the synodic frequency or their harmonics. In this way, the orbital paths of satellites experiencing only forced motion make a family of nested orbits that never intersect.

In contrast to the forced eccentricity, the free eccentricity and inclination are both associated with precession. This effect is a direct result of the fact that the time-averaged potential around a binary does not fall off as $1 / R$ - the binary's mass averaged over its orbit is akin to an oblate spheroid (Heppenheimer 1978; Murray \& Dermott 1999; Lee \& Peale 2006). The precession rates of the periastron and ascending node are

$$
\begin{aligned}
\dot{\varpi} & =\Omega_{\mathrm{g}}-\kappa_{\mathrm{e}} \approx \frac{3}{4} \frac{a_{\mathrm{bin}}{ }^{2}}{R_{\mathrm{g}}{ }^{2}} \frac{\mu}{M} \sqrt{G M / R_{\mathrm{g}}{ }^{3}} \\
\dot{\Omega}_{\text {node }} & =\Omega_{\mathrm{g}}-\nu_{\mathrm{i}} \approx-\frac{3}{4} \frac{a_{\mathrm{bin}}{ }^{2}}{{R_{\mathrm{g}}}^{2}} \frac{\mu}{M} \sqrt{G M / R_{\mathrm{g}}}
\end{aligned}
$$

and thus are approximately equal and opposite. 


\subsection{Incorporating a gaseous disk}

The orbit solutions in Equations (21) - (23) apply directly to non-interacting particles in a disk with negligible mass. Now we consider how gravity, pressure support, and aerodynamic drag from the gas disk modifies these orbits. To make headway, we assume that outside of the critical radius where orbits are unstable, the binary's gravitational potential changes the fluid flow in a disk from circular orbits to most circular paths, similar to the satellite orbits described above, but dependent on the physics of the disk. Our premise is that fluid flow in a gas disk generally tends to circularize, an effect that is enhanced by apsidal precession

of free eccentric orbits (see discussions in Lin \& Pringle 1976, Sver \& Clarke 1992, Ogilvie 2001, Ogilvie \& Barker 2014, and Barker \& Ogilvie 2014). Thus nested, non-intersecting, most circular streamlines are a natural extension to circular flows around a point mass.

Continuing with this picture, we make the simplifying assumption that to a good approximation the disk potential and gas pressure are axisymmetric. If gas fluid elements travel on most circular orbits, then radial and azimuthal variations in disk properties must exist. However, they may be small; in the Kepler circumbinary systems, the forced eccentricities are $e_{\text {force }} \sim 0.002-0.044$ (see $\$ 5$ ). Analytical models confirm that close to the binary, the gravitational effects stemming from disk eccentricity are small compared with the stars' influence on circumbinary orbits. In this way, we build on previous descriptions of circumbinary disks that assert strict axisymmetry in the disk by having fluid elements travel in pressure-supported circular orbits (e.g., Marzari \& Scholl 2000; Scholl et al. 2007).

In the limit of a tenuous gas, this simple picture yields reasonable results. As pressure and disk gravity diminish, gas molecules damp to the same set of most circular orbits derived for satellites in \$2.1. However, the model does not incorporate hydrodynamical effects, including viscosity, turbulence and gravitational instabilities, that may significantly modify disk structure (e.g., Pelupessy \& Portegies Zwart 2013). With this acknowledgement of the limitations of model, we proceed to consider circumbinary orbits.

\subsubsection{Orbits in the presence of a massive disk}

The gravity of a massive circumbinary disk modifies the orbits of satellites around a binary star. An axisymmetric disk, with a gravitational potential $\Phi_{d}(R)$ in the orbital plane of the binary, modifies the mean motion and the epicyclic frequencies of a circumbinary satellite. These properties of the satellite are related to derivatives of the time-average potential; we derive them by making the substitution

$$
\Phi_{00} \rightarrow \Phi_{00}+\Phi_{d}
$$


in Equations (17) and (18). These changes to $\Omega_{\mathrm{g}}$ and $\kappa_{\mathrm{e}}$ cause only minor adjustments to most terms in the orbit solutions except for the term associated with forced eccentricity:

$$
\tilde{C}_{1}^{-}=\frac{1}{\left(\kappa_{\mathrm{e}}^{2}-\Omega_{\mathrm{g}}{ }^{2}\right)}\left[-\frac{d}{d R}\left(\Phi_{01}+\frac{1}{2} \Phi_{01}^{e}\right)+\frac{1}{R}\left(2 \Phi_{01}-\Phi_{01}^{e}\right)\right]_{R_{\mathrm{g}}} .
$$

With $\Omega_{\mathrm{g}}$ and $\kappa_{\mathrm{e}}$ in the denominator, the magnitude of the forced eccentricity now has a wide range of values that depend on the form of the disk potential. In plausible astrophysical conditions, Rafikov (2013) demonstrates that the denominator can be negative and large compared to a disk-free system. The magnitude of the forced eccentricity then becomes small; the forced eccentric orbit is anti-aligned with the binary.

When the denominator in Equation (36) goes to zero, satellite orbits experience a secular resonance (Rafikov 2013). Physically, this condition occurs when orbits with free eccentricity do not precess; contributions to the apsidal precession from the disk and from the binary are equal and opposite. To quantify this behavior, we choose a disk surface density of

$$
\Sigma_{g}(a)=\Sigma_{g, 0} \frac{a_{0}}{a}
$$

with $\Sigma_{g, 0}=2000 \mathrm{~g} / \mathrm{cm}^{2}$ and $a_{0}=1$ AU, typical of observed gas disks (e.g., Dent et al. 2013). The disk potential is

$$
\Phi_{d}=2 \pi G \Sigma_{g, 0} a_{0} \log \left(a / a_{0}\right)
$$

(Bromley \& Kenyon 2011a, Appendix A). The corresponding apsidal precession rate from the disk is

$$
\dot{\varpi}_{d} \approx-\left[\frac{1}{2 \Omega_{\mathrm{g}} R^{2}} \frac{d}{d R}\left(R^{2} \frac{d \Phi_{d}}{d R}\right)\right]_{R_{\mathrm{g}}}=-\frac{\pi}{\Omega_{\mathrm{g}} R_{\mathrm{g}}} G \Sigma\left(R_{\mathrm{g}}\right) .
$$

Setting the magnitude of this expression equal to the apsidal precession rate from the binary (Equation (33) ) yields the radial position of the resonance.

For the Kepler circumbinary planets, the resonance lies at an orbital distance of roughly 2 AU. This distance is between the orbits of the known planets (Rafikov 2013) and the likely position of the snow line (Kennedy \& Kenyon 2008). As the disk dissipates, however, the resonance sweeps outwards past the snow line. This motion may have interesting implications for the formation and inward migration of gas giants 3

\footnotetext{
${ }^{3}$ It is beyond our scope to treat the orbital dynamics at the resonance in detail. Aside from its impact on the orbits of solids, it is unclear how the gas reacts. Within the Lee-Peale-Leung theory, the evolution equation for the $\tilde{C}_{1}^{-}$mode on resonance is an undamped, driven harmonic oscillator with a natural frequency of $\Omega_{\mathrm{g}}$. This solution predicts a linear growth time scale for eccentricity of $10^{3}-10^{4}$ years for the Kepler circumbinary planetary systems, which may be an overestimate (Meschiari 2014, Figure 6).
} 
An extension to the axisymmetric form for the disk potential would account for the non-axisymmetric ebb and flow of fluid elements on most circular orbits. A starting point is to approximate the streamlines with the forced eccentric orbits from secular perturbation theory (e.g., Silsbee \& Rafikov 2015b,a). The gravitational field of the eccentric disk then affects the forced eccentricity, but not the apsidal alignment (see Equations (19)-(21) in Silsbee \& Rafikov 2015a). We could then calculate a self-consistent value for $e_{\text {force }}$ where the orbits of the fluid elements follow paths that they help to generate. Further extensions to the theory of eccentric orbits would include the effects of geometric compression of fluid density from the continuity equation and related hydrodynamical effects for flow along most circular paths (e.g., Ogilvie \& Barker 2014; Barker \& Ogilvie 2014).

\subsubsection{Orbits in the presence of a pressurized disk}

The pressure in protoplanetary gas disks modifies the orbit of any fluid element. In a simple axisymmetric circumstellar disk where the radial pressure gradient is positive, pressure support leads to sub-Keplerian orbits (e.g., Weidenschilling 1977a; Birnstiel et al. 2010; Chiang \& Youdin 2010). The mean orbital speed of the gas relative to a circular Keplerian orbit is

$$
|\Delta v| \approx \eta R_{\mathrm{g}} \Omega_{\mathrm{g}}
$$

where $\eta \sim 10^{-3}$ (e.g., Weidenschilling 1977a). In a circumbinary disk, we estimate the effect of pressure by assuming that the pressure gradient is radial, and treating $\eta$ as a constant, independent of orbital position, at least in some local region of the disk. We then may let pressure support appear in the equations of motion as a change in the total mass of the binary:

$$
\Phi_{00} \rightarrow(1-2 \eta) \Phi_{00}
$$

leading to small modifications in quantities including $\Omega_{\mathrm{g}}$ and $\kappa_{\mathrm{e}}$. Thus, gas pressure increases the forced eccentricity by a factor of $1+2 \eta$ compared to an unpressurized disk.

In this approximation, the gas and solids not coupled to it are on distinct most circular orbits (Thébault et al. 2006; Meschiari 2014; Silsbee \& Rafikov 2015a). Nonetheless, the forced eccentric orbits of disks with and without pressure support are apsidally aligned. Furthermore the differences between forced eccentric paths of gas and the uncoupled solids are small. They lead to relative radial speeds between gas and these solids of

$$
\Delta v_{r} \sim \eta e_{\text {force }} v_{\mathrm{K}}
$$

which, for anticipated values of $e_{\text {force }}$ for the Kepler circumbinary planets, is roughly two orders of magnitude smaller than the "headwind" felt by the solids as they plow through 
the gas. In other words, the most circular paths associated with pressure-supported gas and uncoupled solids are sufficiently similar that relative velocities arise predominantly from their azimuthal motion, just as in circumstellar disks.

\subsubsection{Gas drag}

When the gaseous disk has internal pressure, material orbits the central object more slowly than the solids. The solids then experience aerodynamic drag (Adachi et al. 1976; Weidenschilling 1977a). Particles smaller than about a centimeter are fully entrained in the gas. Planetesimals with radii of $1 \mathrm{~km}$ or more barely feel the gas. Both sets of particles follow distinct most circular orbits. For intermediate particle sizes, aerodynamic drag slows azimuthal speeds. Without the benefit of radial pressure support, these objects spiral inward (if the gas is sub-Keplerian). The inspiral time scales are as fast as $1 / \eta$ dynamical times (Adachi et al. 1976; Weidenschilling 1977a; Weidenschilling \& Cuzzi 1993; Chiang \& Youdin 2010; Youdin \& Kenvon 2013).

To describe the dynamics of a particle experiencing gas drag, we take advantage of the small difference between the forced eccentricities of a pressurized gas disk and satellites that orbit in the absence of gas drag. Approximating the effect of gas as a constant azimuthal headwind, the equations of motion resolve to a most circular orbit with some intermediate

forced eccentricity and a small amount of radial drift (e.g., Youdin \& Kenyon 2013). Importantly, if the epicyclic motion of the entrained particles and large solids are apsidally aligned, then so are the epicyclic orbits of these intermediate-size bodies. We confirm below using numerical tests that this description is reasonable (\$3).

\subsection{Significance for circumbinary planet formation}

Here, we highlight several features of the orbit solutions described in $\$ 2.1$ and modifications arising from the presence of a gas disk (\$2.2):

- The forced epicyclic motion (the $\tilde{C}_{1}^{-}$term) is synchronized with the guiding center. The addition of an axisymmetric potential, whether it describes the effects of a massive disk or mimics the behavior of radial pressure, preserves this relationship. In general, forced eccentric orbits remain apsidally aligned with the binary. In a disk free of gas pressure, particles on these paths never collide.

- Pressure support changes the most circular paths of the gas streamlines relative to the 
orbits of solid particles not susceptible to aerodynamic drag. This behavior generates relative epicyclic motion between the gas and the solids. If the forced eccentric orbits are apsidally aligned with the binary, however, then the relative speeds induced by the differences in the most circular paths are much smaller that the usual headwind felt by the solids plowing through the gas.

- The forced epicyclic motion is coupled in its alignment with the binary. Thus if the binary itself precesses slowly, then the satellite's argument of periapse also precesses. To visualize this point, we note that a slowly precessing binary orbit has slightly different radial and azimuthal frequencies. To the solutions of Leung \& Lee (2013), we thus add the precession rate $\dot{\varpi}_{\text {bin }}$ to the binary's orbital frequency, $\Omega_{\text {bin }}$, without modifying the synodic frequency $\omega_{\text {syn }}$ between satellite and binary. The terms most greatly affected by this small change are the ones associated with the forced eccentricity (e.g., with $\tilde{C}_{1}^{-}$). The time dependence in these terms then transforms as

$$
\cos \left(\omega_{\mathrm{syn}} t-\Omega_{\mathrm{bin}} t\right) \rightarrow \cos \left(\left(\Omega_{\mathrm{g}}+\dot{\varpi}_{\mathrm{bin}}\right) t\right)
$$

Thus the satellite's forced epicyclic motion precesses with the binary, remaining apsidally aligned (in secular resonance).

These properties may be essential for circumbinary planet formation. They suggest that most circular orbits remain nested and non-intersecting even in the presence of a massive disk or if the binary precesses due to interactions with a massive disk or a distant planetary/stellar perturber. Gas dynamics add complications, although if a protoplanetary gas disk is nearly axisymmetric and streamlines follow most circular orbits, then the presence of the binary induces only small additional relative speeds compared to the circumbinary case. Thus issues of entrainment, gas drag, and the "one-meter barrier" from circumstellar planet formation (e.g., Youdin \& Chiang 2004; Birnstiel et al. 2010; Chiang \& Youdin 2010; Windmark et al. 2012; Garaud et al. 2013; Youdin \& Kenyon 2013) carry over to the circumbinary environment.

Other studies of circumbinary planet formation include disks that are exactly axisymmetric and circular in their orbital flow (e.g., Scholl et al. 2007) or that are neither axisymmetric or apsidally aligned with the binary (Silsbee \& Rafikov 2015a). In these cases, aerodynamic drag on particles leads them to achieve orbits that depend on their physical size. In misaligned disks, for example, the magnitude of the forced eccentricity and the apsidal orientation of forced eccentric orbits depend on particle size (Silsbee \& Rafikov 2015a). In either case, particles of different size experience high-speed, destructive collisions (Marzari \& Scholl 2000; Thébault et al. 2006). However, gas disks are strongly dissipative. 
We assume that most circular orbits, aligned with the binary, are a good first approximation to the paths of fluid elements. In any event, as the gas dissipates, we expect that the fluid elements and solids are likely to settle on the same set of most circular orbits.

\subsection{Summary of the analytical theory}

The analytic theory of circumbinary orbits predicts a family of nested most circular paths. Satellites orbiting with these paths (i) make well-defined minimal radial excursions and (ii) never collide. Thus, these paths define "dynamically cold" orbits in exactly the same way as circular orbits around single stars. As in the Keplerian case, satellites may have additional "free" eccentricity and inclination to describe motion about these paths. For any combination of free and forced eccentricity, the dynamics of planetesimals as they stir or damp each other takes place in the frame of these most circular paths.

While the physics of gaseous protoplanetary disks is uncertain, gas fluid elements can follow most circular paths, even when the disk mass and pressure support are significant. These orbits serve as reference frames for local hydro- and aerodynamics. We expect that gas settles to these orbits, since they allow for streamlines to be nested and non-crossing with minimal radial excursions, despite any forced eccentric motion. Studies of eccentric gas disks around

a single star (Syer \& Clarke 1992; Ogilvie 2001; Ogilvie \& Barker 2014; Barker \& Ogilvie 2014) highlight potential differences between the hydrodynamics of circular flows and eccentric ones, including mass conservation along eccentric streamlines (Ogilvie \& Barker 2014; Barker \& Ogilvie 2014). In circumstellar disks, gas might find stable orbits with some free eccentricity (Syer \& Clarke 1992). However, it seems more likely that apsidal precession circularizes these orbits (Ogilvie 2001). For circumbinary disks, we expect that apsidal precession leads to fluid flow along most circular paths.

\subsection{Limitations of the theory: resonances and chaos}

Limitations of the theory stem from its perturbative approach, particularly that it is linear in eccentricities. Aside from singularities in the coefficients in the solutions (e.g., $C_{k}$, $\tilde{C}_{k}^{ \pm}$) at the 1:1, 1:2 and 1:3 commensurabilities - along with the possible resonance induced by a massive disk (Rafikov 2013, see \$2.2.1, above) - the theory does not accommodate resonant effects (Wisdom 1982, 1983; Lecar et al. 2001). These phenomena would arise in the equations of motion if the satellite's eccentric motion were explicitly included in the calculation of the force. Then, solutions may become chaotic and possibly unstable (Wisdom 
1980).

The formula for $a_{\text {crit }}$ provides a starting point for investigating instability (Equation 1, Holman \& Wiegert 1999). In this approximation, the location of unstable orbits close to the binary varies smoothly with the mass ratio and binary eccentricity. However, locating all of the unstable orbits is more complicated (Musielak et al. 2005; Doolin \& Blundell 2011; Chavez et al. 2015). Overlapping resonance conditions generate instability (Chirikov 1959, 1979; Wisdom 1980); thus, unstable orbits can exist in narrow, isolated ranges of orbital distance $a$ beyond $a_{\text {crit }}$. In the binary Kepler-16 (Table 1), the 5:1 resonance, located outside of $a_{\text {crit }}$, is unstable. Although it is weaker, the 6:1 resonance is also unstable (e.g., Pierens \& Nelson 2007). In between these resonances, orbits are stable; Kepler-16b resides on one of these stable orbits (Popova \& Shevchenko 2013; Chavez et al. 2015). Beyond the $6: 1$ resonances, all orbits are stable for small planetary eccentricities. When we discuss formation mechanisms for circumbinary planets, we return to this issue (\$5).

\section{Numerical simulations}

The analytic theory for circumbinary orbits is first-order accurate in the binary eccentricity and is derived from equations of motion linearized in the excursions away from the guiding center. Despite these limitations, it compares well in numerical experiments even when the binary eccentricity is moderately large. We summarize these experiments in this section.

\subsection{Code description}

We apply our planet formation code OrCHESTRA (Bromley \& Kenvon 2006, 2011a) to compare with the theoretical results in $\$ 2$. ORCHESTRA is a hybrid $n$-body-coagulation code for tracking the emergence of individual planets ( $n$-bodies) from a sea of smaller particles that can be characterized statistically (the coagulation "grid" with bins for radial position and particle mass). In a standard hybrid calculation, small particles within a set of concentric annuli begin with an adopted radial surface density and initial $e$ and $i$ relative to a circular orbit (see also Safronov 1969; Lissauer 1987; Spaute et al. 1991; Wetherill \& Stewart 1993; Weidenschilling 1989; Kenyon \& Luu 1998; Kenyon \& Bromley 2008). Initially, there are no $n$-bodies. As massive objects evolve in the grid, the most massive are "promoted" into the $n$-body part of the code. The subsequent evolution of the $n$-bodies and the grid are linked together, enabling the simultaneous tracking of gravitational interactions and collisions that 
lead to accretion, merging and fragmentation (e.g., Kenyon \& Bromley 2006, 2010, 2014).

ORCHESTRA has other capabilities which allow us to consider a variety of problems in planet formation and evolution. It can track a swarm of massive or massless tracer particles usually sampled from the orbital distribution of the coagulation particles - to mediate interactions that involve resonances and migration (Bromley \& Kenyon 2011b). The code also includes interactions with a massive gas disk, both through gravity (Bromlev \& Kenyon 2011a) and aerodynamic drag (Weidenschilling 1977a; Kenyon \& Bromley 2002). Tests of these and other elements of the code, including our choice of time integrator - either symplectic (Yoshida 1990) or adaptive Richardson extrapolation at $6^{\text {th }}$-order (Bromlev \& Kenvon 2006) - are summarized in Kenvon \& Bromley (2001), Bromley \& Kenyon (2006), Bromley \& Kenvon (2011a), and Kenyon \& Bromley (2015).

Here we use the $n$-body component of the code with tracer particles to calculate orbits around a binary. The primary and secondary stars are $n$-bodies, evolved with the $6^{\text {th }}$-order symplectic integrator. Energy errors are better than one part in $10^{10}$. In some runs (see below) we include a third, Jupiter-mass $n$-body as a perturber. In others we include a massive gas disk, choosing a surface density $\Sigma$ in Equation (37) and the corresponding gravitational potential in Equation (38) to use in the equations of motion for the tracers. This approach allows us to test the analytic theory in $\$ 2$ using orbit solutions derived numerically at high accuracy.

\subsection{Simulation results}

Simple $n$-body experiments with ORCHESTRA allow us to test several key features and predictions of the analytic theory ( $\S 2$ ). For these studies, we use the Kepler-16 system as an example (Dovle et al. 2011), adopting binary parameters in Table 1 as estimated by Leung \& Lee (2013).

We begin with several illustrations of circumbinary orbits with no other massive perturbers. Our first example focuses on most circular orbits, showing both high-frequency oscillations and epicyclic motion. In a second example, we consider the difference between the time evolution of particles on most circular and 'initially' circular orbits around a eccentric binary. Particles on most circular orbits do not precess. Particles which start out on geometrically circular orbits have some free eccentricity; this component of the orbit precesses. By selecting an initially circular orbit with equal parts of free and forced eccentricity, we show how the precession of the free eccentric orbit modifies trajectories around the binary.

This second example identifies issues with previous $n$-body studies of circumbinary 
planet formation (e.g., Moriwaki \& Nakagawa 2004; Scholl et al. 2007; Meschiari 2012; Paardekooper et al. 2012). In these analyses, initially geometrically circular orbits precess, causing high relative collision velocities that can inhibit the growth of planetesimals into planets. Most-circular orbits have smaller radial excursions and provide a calmer frame of reference for planet formation.

We then explore the impact of a massive disk, gas drag, and a Jupiter-mass perturber. Our goal is to investigate whether most circular orbits remain "most circular" in the context of a protoplanetary disk around a binary star.

- A sequence of most circular orbits. Figure 1 shows the result of test particles on most circular paths near the orbital distance of Kepler-16b. In these orbits, most of the motion comes from the forced eccentricity. As suggested by the Figure, the orbits are all nested and do not intersect.

- A most circular orbit in detail. Figure 2 contains radial excursions of a satellite of Kepler-16 where the binary's eccentricity is reduced by a factor of ten. With this low eccentricity, both the high-frequency driven oscillations and the forced eccentric orbit are apparent. This Figure shows a direct comparison to theory (Equation (21)).

- "Circular" versus Most-circular. Satellites can be launched on paths that are initially more circular (smaller radial excursions) than the most circular orbits discussed here. However, these satellites do not remain on circular paths as their orbits evolve over time. Figure 3 illustrates this point. For a central binary with non-zero $e$, an orbit with equal parts of free and forced eccentricity can be initialized on a purely circular orbit about the binary center-of-mass. At the start (time $=0$ in the figure), this orbit has very little epicyclic motion (e.g., $\delta R$ is close to zero). Over time, the free eccentric orbit precesses (Equation (33)); the forced eccentric orbit does not. Hence the two modes drift in phase, causing the beat pattern in the radial excursion shown in the Figure. Thus, orbits can be geometrically "circular" but only temporarily. Most-circular orbits have the smallest radial excursions over the long term.

Previous studies, including our own (Kenyon \& Bromley 2014), describe simulations to track particle dynamics around binaries. If particles are set up initially on geometrically circular orbits (where free and forced eccentricities cancel at $t=0$ ), they appear to experience "secular excitations" where the total eccentricity increases with time until $e=2 e_{\text {force }}$ (Moriwaki \& Nakagawa 2004; Scholl et al. 2007; Meschiari 2012; Paardekooper et al. 2012; Rafikov 2013). However, the particles are never actually stirred by the binary. They simply experience the independent free and forced modes of epicyclic oscillation, acting in concert but not in phase. 
- Most-circular paths around a high-eccentricity binary. In Figure 4, the eccentricity of the Kepler-16 binary is increased to $e_{\mathrm{bin}}=0.5$. The satellite is at $0.9 \mathrm{AU}$, just outside the critical radius for stable orbits for this choice of $e_{\mathrm{bin}}$. The Figure shows samples of the planet's position in the plane of the binary, taken over the course of $10^{4}$ satellite orbits (blue points in the Figure). The samples reveal the forced eccentricity and demonstrate that the periastron of the satellite is fixed and aligned with the binary. This numerical test is significant: the analytic theory described here is linear, but the $n$-body experiment is not. The orbital alignment - a prediction of the linear analytic theory — holds in the non-linear case.

- Orbits in a massive disk. Figure 4 also demonstrates that most circular orbits do not precess even in the presence of a massive disk. We use a potential as in Equation (37) with a surface density of $2000 \mathrm{~g} / \mathrm{cm}^{2}$ at 1 AU. Around a single star with Kepler-16's mass, the disk causes rapid apsidal precession of a satellite at $0.9 \mathrm{AU}, \dot{\varpi} \sim 0.005 \mathrm{yr}^{-1}$ (Rafikov 2013). However, around the binary, the satellite's forced eccentric orbit is immune to precession from the disk, as we expect from perturbation theory. The example in the Figure demonstrates that this prediction extends to moderately large values of the binary's eccentricity.

- Gas drag. Aside from changing the gravitational potential, gas modifies the orbits of small solids through aerodynamic drag. Figure 5 shows a particle orbit in a subKeplerian ("pressurized") gas disk whose mean azimuthal speed is reduced by a factor of $1-\eta$ relative to a guiding center orbit in the disk's absence $(\eta=0.001$; Equation (40)). For the purpose of this illustration, we do not include the disk's gravity. The drag force is proportional to the particle's speed in the local most circular reference frame of the gas. The magnitude of the force is sufficient to cause the particle to inspiral within a few hundred orbital periods. The Figure illustrates that even in the presence of gas drag, the particle orbits remain aligned with the binary.

- Effects of stirring: external time-dependent perturbations. Collisional damping drives particles toward most circular orbits because particles coexist on these orbits without collisions. Conversely, distant massive perturbers (i.e., planets) gravitationally stir particles, driving them away from most circular paths. From the analytic theory, we expect stirring to behave the same way around a binary as it does around a single star. Figure [ ] shows simulation data from three scenarios: (i) a satellite like Kepler-16b; (ii) a satellite together with a Jupiter mass-body on a circular orbit at 2 AU; and (iii) a satellite and a Jupiter orbiting a central point mass. Perturbations of the more distant planet affect the motion of satellites in circumstellar and circumbinary cases in much the same way, by generating nearly identical free eccentricity. 
- Binary precession. When an eccentric binary precesses, we expect the satellite's forced eccentric orbit apsidally precesses at the same rate. Figure 7 illustrates this behavior. In a reference frame rotating with a precessing eccentric $\left(e_{\text {bin }}=0.5\right)$ binary, the satellite's orbit shows no precession due to a Jupiter mass perturber at 2 AU.

\subsection{Summary of the numerical studies}

To summarize, this set of numerical experiments illustrates that the main predictions of the linear analytic theory are confirmed in the non-linear regime. A key result is that particles initialized on geometrically circular Keplerian orbits have a free eccentricity, which leads to precession and possibly high-velocity collisions among particles on adjacent orbits. In contrast, most circular orbits remain nested and never cross, even in the presence of a massive disk or a gas giant perturber. Our conclusion is that the standard initial conditions of particles on geometrically circular orbits are not realistic in a dynamically cool planetary disk. The nested most circular orbits are a better starting point. In the next section, we examine these conclusions in the context of planet formation theory.

\section{Circumbinary planet formation}

In the standard theory of star and planet formation, a rotating molecular cloud of gas and dust collapses into a central protostar and a circumstellar disk (e.g., Cassen \& Moosman 1981; Terebev et al. 1984; Yorke et al. 1993). Disk material is on nearly circular orbits (e.g., Weidenschilling 1977a); viscous shear transports mass inward and angular momentum outward (Lvnden-Bell \& Pringle 1974). Small solids are well-coupled to and flow with the gas (e.g., Adachi et al. 1976; Weidenschilling 1977a; Rafikov 2004; Birnstiel et al. 2010; Chiang \& Youdin 2010). Larger solids decouple from the gas and follow Keplerian orbits about the central star.

Among the decoupled solids, various dynamical processes tend to circularize their orbits around the central star. Smaller particles feel a strong headwind and are dragged towards the central star. Although larger particles feel less drag, the gas efficiently damps their orbits (see also Safronov 1969; Greenberg et al. 1978; Spaute et al. 1991; Wetherill \& Stewart 1993). Along with gas drag, collisional damping and dynamical friction drive particles towards circular orbits (e.g., Hornung et al. 1985; Wetherill \& Stewart 1993; Kenyon \& Luu 1998; Goldreich et al. 2004). As a result, most particles experience small relative collision velocities which encourages growth through mergers (see also Youdin \& Kenyon 2013). 
When the central protostar is a close binary, we expect a similar evolution. Infalling gas forms a circumbinary disk where the gas and dust generally follow most circular paths around the binary. Aside from an inner gap in the disk roughly at $a_{\text {crit }}$, the structure of the disk orbiting a circular binary is fairly similar to a circumstellar disk around a single star (e.g., Lin \& Papaloizou 1979; Pringle 1991; Artymowicz \& Lubow 1996). For eccentric binaries, disk material appears to follow most circular orbits with forced eccentricity, driven by the central binary (e.g., Pierens \& Nelson 2007; Pelupessy \& Portegies Zwart 2013). As long as the disk is not dominated by non-axisymmetric structure (e.g., spiral density waves in a massive disk), we expect pressure and viscosity to induce a smaller inward drift of the gas

and small particles relative to most circular orbits (see also Pichardo et al. 2005, 2008). The gas attempts to circularize the orbits of larger particles onto most circular orbits. Collisional damping and dynamical friction also damp the orbits. Thus, large particles end up on most circular orbits with a small amount of free eccentricity with a magnitude similar to the eccentricity of particles in disks around a single star.

Achieving these configurations is a natural long-term outcome for dissipative disks. However, it is worth considering how quickly the gas, dust, and larger solid particles circularize. We then compare these time scales with time scales for other processes such as precession of the binary. We expect that particles settle onto most circular orbits when the precession time scales are long relative to circularization time scales. Here, we consider several basic time scales, treating gas-dominated and particle disks as separate cases due to differences in their surface densities and in the physical processes that drive them.

\subsection{Gas disks}

We start by considering a massive circumbinary disk to model primordial gas in protoplanetary systems (Weidenschilling 1977b; Hayashi 1981). As before, we choose a surface density

$$
\Sigma_{g}(a)=\Sigma_{g, 0}\left(\frac{a}{a_{0}}\right)^{-1}
$$

where $\Sigma_{g, 0}=2000 \mathrm{~g} / \mathrm{cm}^{2}$, and $a_{0}=1$ AU. The disk has a vertical scale height

$$
h(a)=h_{g, 0}\left(\frac{a}{a_{0}}\right)^{q}
$$

where $h_{g, 0} / a_{0} \sim 0.02$ and $q=9 / 7$ (Chiang \& Goldreich 1997).

Damping times in this disk derive from the sound speed,

$$
c_{s}(a) \sim h(a) v_{\mathrm{K}} / a,
$$


where $v_{\mathrm{K}}$ is the orbital velocity at distance $a$. The time scale for vertical structures to reach hydrostatic equilibrium is $h / c_{s}$, close to the dynamical time (Lightman 1974; Pringle 1981). Pressure damping of features along an orbital path require at least

$$
T_{g, \text { damp }} \gtrsim a / c_{s} \sim \frac{a}{h} T_{\mathrm{K}} \approx 8\left[\frac{a}{1 \mathrm{AU}}\right]^{17 / 14}\left[\frac{M}{1 \mathrm{M}_{\odot}}\right]^{-1 / 2} \mathrm{yr} .
$$

where $M$ is the total central mass. Radial structures may dissipate more slowly, on a diffusive time scale (e.g., Ogilvie 2001)

$$
T_{g, \text { damp }} \lesssim \frac{a^{2}}{h^{2}} T_{\mathrm{K}} \approx 2.5 \times 10^{3}\left[\frac{a}{1 \mathrm{AU}}\right]^{13 / 14}\left[\frac{M}{1 \mathrm{M}_{\odot}}\right]^{-1 / 2} \mathrm{yr} .
$$

We anticipate that disks with free eccentricity damp to most circular orbits on time scales in this range.

By comparison, the time scale for binary precession, a result of the gravitational interaction between the gas disk and the binary, is long. If the inner edge of the disk is at orbital distance $a_{\text {in }}$, then the binary's apsidal precession rate is (e.g., Ward 1981; Rafikov 2013):

$$
\dot{\varpi}_{\mathrm{bin}} \approx 0.5 \frac{\pi G \Sigma_{g}\left(a_{\mathrm{in}}\right)}{\Omega_{\mathrm{bin}} a_{\mathrm{in}}}\left(\frac{a_{\mathrm{bin}}}{a_{\mathrm{in}}}\right) .
$$

The precession time is then

$$
\left.T_{\text {bin-pre }} \approx 2.0 \times 10^{4}\left[\frac{\Sigma_{g, 0}}{2000 \mathrm{~g} / \mathrm{cm}^{2}}\right]^{-1}\left[\frac{a_{\text {bin }}}{0.2 \mathrm{AU}}\right]^{1 / 2}\left[\frac{a_{\text {in }}}{2.5 a_{\text {bin }}}\right]^{3} \text { yr } \quad \text { [gas disk }\right],
$$

where we choose to set $a_{\text {in }}$ to be the innermost stable orbit at $a_{\text {crit }} \approx 2.5 a_{\text {bin }}$, for the values of the parameters in the angular brackets.

Thus, binary precession is slow compared with dynamical times. Precession is a perturbative effect. We therefore expect that orbits in the disk remain apsidally aligned with the binary. We conclude that gas disks damp quickly to most circular orbits, so long as the physics (gravity, hydrodynamics) enables the disk to be axisymmetric when averaged over these orbits (although see Pelupessy \& Portegies Zwart 2013).

\subsection{Particle disks}

As the gaseous disk evolves, solids particles evolve with it. Small particles remain coupled to the gas. Larger particles with sizes of $1 \mathrm{~cm}$ or larger (depending on the local

properties of the gas) are uncoupled (e.g., Adachi et al. 1976; Weidenschilling 1977a; Rafikov 
2004; Birnstiel et al. 2010). Collisional processes enable some particles to grow to larger sizes (e.g., Windmark et al. 2012; Garaud et al. 2013).

For large particles, interactions with the gas produce a radial drift and circularize the orbits. The characteristic time scale for these interactions is (Adachi et al. 1976; Weidenschilling 1977a; Chiang \& Youdin 2010):

$$
T_{\text {circ }} \approx 15\left(\frac{r}{1 \mathrm{~km}}\right)\left(\frac{1 \mathrm{AU}}{a}\right)\left(\frac{10^{-9} \mathrm{~g} \mathrm{~cm}^{-3}}{\rho_{\mathrm{g}}}\right) T_{\mathrm{K}},
$$

where $r$ is the radius of the particle, $\rho_{g}$ is the local gas volume density (normalized to the typical density of a minimum mass solar nebula), and $T_{\mathrm{K}}$ is the orbital period of the local guiding center. For all but very large objects with $r \gtrsim 100 \mathrm{~km}$, this time scale is small compared with the precession time. Thus, small particles uncoupled from the gas likely find most circular orbits.

Numerical simulations of ensembles of small particles interacting with the gas suggest the surface density of the solids is somewhat steeper than the surface density of the gas (e.g., Youdin \& Chiang 2004; Brauer et al. 2008; Birnstiel et al. 2010, 2012; Laibe et al. 2012; Pinte \& Laibe 2014). Here we adopt a standard power law:

$$
\Sigma(a)=\Sigma_{0}\left(\frac{a}{a_{0}}\right)^{-1.5}
$$

where $\Sigma_{0}=10 \mathrm{~g} / \mathrm{cm}^{2}$. To derive basic time scales, we assume that particles in the disk have radii of $r=1 \mathrm{~km}$ and a density of $3 \mathrm{~g} / \mathrm{cm}^{3}$. If they are stirred to their escape velocity,

$$
\begin{aligned}
v_{\mathrm{esc}} & =\sqrt{2 G m / r}=\sqrt{(8 \pi G \rho / 3)} r \\
& =130\left[\frac{\rho}{3 \mathrm{~g} / \mathrm{cm}^{3}}\right]^{1 / 2}\left[\frac{r}{1 \mathrm{~km}}\right] \mathrm{cm} / \mathrm{s},
\end{aligned}
$$

then we can use standard kinetic theory to estimate the collision time as $(n \sigma v)^{-1}$ where $n$ is the number density, $\sigma$ is the collisional cross-section, and $v$ is the relative velocity. The damping time from collision is then

$$
\begin{aligned}
T_{\text {damp }} & \sim \frac{\rho r T_{\mathrm{K}}}{2 \sqrt{2} \pi \Sigma} \\
& \approx 3400\left[\frac{\rho}{3 \mathrm{~g} / \mathrm{cm}^{3}}\right]\left[\frac{r}{1 \mathrm{~km}}\right]\left[\frac{a}{1 \mathrm{AU}}\right]^{6}\left[\frac{\Sigma_{0}}{10 \mathrm{~g} / \mathrm{cm}^{2}}\right]^{-1}\left[\frac{M}{1 \mathrm{M}_{\odot}}\right]^{-1 / 2} \mathrm{yr} .
\end{aligned}
$$

where we assume that collisions are inelastic and the disk scale height is proportional to the relative speed $v$, divided by the orbital frequency. This expression ignores gravitational focusing, which reduces the damping time (e.g., Wetherill \& Stewart 1993; Ohtsuki 1999). 
While the damping time is only linearly dependent on particle size, the range of sizes can be considerable in an evolving planet-forming disk. For pebbles $(r \approx 1 \mathrm{~cm})$, the damping time is very fast: $T_{\text {damp }}$ is formally shorter than a dynamical time. For large particles $(\gtrsim 1 \mathrm{~km})$, the time scale for collisional damping is fairly long, approaching the precession time scale for the gas when $r \gtrsim 10 \mathrm{~km}$. However, these larger particles interact with smaller particles through dynamical friction and viscous stirring (e.g., Ohtsuki et al. 2002; Goldreich et al. 2004). For these processes, the typical damping time for large particles is a factor of 10-100 smaller than suggested by Equation (54). Thus, damping times for particles with $r \lesssim 100 \mathrm{~km}$ are short compared with the precession time.

For comparison, the gravity of the particle ring induces the binary to precess with a period

$$
\left.T_{\text {bin-pre }} \approx 4.0 \times 10^{6}\left[\frac{\Sigma_{0}}{10 \mathrm{~g} / \mathrm{cm}^{2}}\right]^{-1}\left[\frac{a_{\text {bin }}}{0.2 \mathrm{AU}}\right]^{1 / 2}\left[\frac{a_{\text {in }}}{2.5 a_{\text {bin }}}\right]^{3} \text { yr } \quad \text { [particle disk }\right] .
$$

We conclude that in a particle disk, as in a gas disk, conditions allow for damping to most circular orbits, so they may serve as the equivalent to circular Keplerian orbits.

Eventually, the largest particles contain more than half the mass of all the solids in an annulus of the disk. At this point, damping times become much longer than stirring times;

orbital evolution then becomes chaotic (e.g., Chambers \& Wetherill 1998; Goldreich et al. 2004; Kenyon \& Bromley 2006). Around a single star, chaotic systems eventually settle down into stable multi-planet systems. Aside from the impact of the inner unstable region and resonances, we expect a similar evolution of chaotic systems around binary stars (e.g., Quintana \& Lissauer 2006).

\subsection{Perturbations from massive planets}

In a particle disk, a circumbinary Jupiter-mass planet dramatically shortens the precession time for the binary. If planetesimals orbit within a few times the binary separation and the massive planet orbits well outside this region (e.g., as in Figure 6, $a_{\text {bin }} \sim 0.2$ AU, satellite at $a \sim 1 \mathrm{AU}$ and "jupiter" at $2 \mathrm{AU}$ ), then the binary precession rate is

$$
\dot{\varpi}_{\mathrm{bin}} \approx \frac{3}{4} \frac{m_{\mathrm{j}}}{M} \frac{a_{\mathrm{bin}}^{3}}{a_{\mathrm{j}}^{3}} \Omega_{\mathrm{bin}}
$$

where $M_{\mathrm{j}}$ and $a_{\mathrm{j}}$ are the planet's mass and orbital distance. Substituting parameter values comparable to observed binary systems and consistent with the set-up in Figure 6, the 
precession period $\left(2 \pi / \dot{\varpi}_{\text {bin }}\right)$ is

$$
T_{\text {bin-pre }} \approx 1.2 \times 10^{5}\left[\frac{M}{1 \mathrm{M}_{\odot}}\right]^{1 / 2}\left[\frac{M}{1 \mathrm{M}_{\mathrm{Jupiter}}}\right]^{-1}\left[\frac{a_{\text {bin }}}{0.2 \mathrm{AU}}\right]^{-1 / 2}\left[\frac{a_{\mathrm{j}}}{2 \mathrm{AU}}\right]^{2} \mathrm{yr} .
$$

Any binary precession from the gas giant perturber is a small perturbative effect that preserves the apsidal alignment between binary and planetesimal orbits.

\subsection{Planet formation in circumbinary disks}

Our analysis establishes time scales for gas and particles to damp to most circular orbits. With no precession of the binary, these orbits do not precess. Although binary precession induces precession in most circular orbits, these orbits remained apsidally aligned with the binary. In this way, most circular orbits provide reference frames in which the dynamics of planet formation takes place (see also Pichardo et al. 2005, 2008). For example, collisional damping and self-stirring of planetesimals modifies the free eccentricity of circumbinary planetesimals (e.g., as in Wetherill 1980); these processes have no impact on the forced eccentricity (and high-frequency modes) driven by the central binary. Gravitational stirring from distant planets (e.g., Weidenschilling 1989) also excites eccentricity, driving epicyclic motion in a manner similar to the binary itself (Heppenheimer 1978, see Figure 6).

When ensembles of orbiting planetesimals attempt to grow into a planetary system, the relative velocity between particles,

$$
v \sim e_{\text {free }} v_{\mathrm{K}}
$$

is a key parameter which establishes the efficiency of gravitational focusing and collision outcomes (Stewart \& Wetherill 1988; Wetherill \& Stewart 1993; Ohtsuki 1999; Ohtsuki et al. 2002). Small relative velocities favor growth by mergers; large relative velocities favor destruction. To estimate the boundary between these regimes, we rely on the specific collision energy $Q_{d}^{*}$ required to disperse half the mass of a colliding pair of planetesimals to infinity. For rocky material,

$$
Q_{d}^{*} \approx 3 \times 10^{5}\left[\frac{r}{1 \mathrm{~km}}\right]^{-0.4}+7 \times 10^{6}\left[\frac{\rho}{3 \mathrm{~g} / \mathrm{cm}^{3}}\right]\left[\frac{r}{1 \mathrm{~km}}\right]^{1.35} \mathrm{erg} / \mathrm{g}
$$

(Davis et al. 1985; Wetherill \& Stewart 1993; Benz \& Asphaug 1999; Housen \& Holsapple 1999; Kenyon \& Bromley 2005). Equating $Q_{d}^{*}$ to the specific rest-frame collision energy between two equal-mass objects, mass loss exceeds mass gain when

$$
v_{\text {dest }} \gtrsim 0.1 \mathrm{~km} / \mathrm{s} \quad \text { [destructive collisions, } r=1 \mathrm{~km} \text { ] }
$$


for kilometer-size rocky planetesimals. This relative speed is best measured in the reference frames of most circular orbits.

Around a binary at orbital distance $a$, all planetesimals have an additional component to their velocity compared with their counterparts around single stars. This velocity, from the forced eccentricity, has a typical magnitude

$$
\begin{aligned}
v \sim v_{\text {force }} & \equiv e_{\text {force }} v_{\mathrm{K}} \approx \frac{\left(M_{p}-M_{s}\right)}{M} \frac{a_{\text {bin }}}{a} e_{\text {bin }} v_{\mathrm{K}} \\
& \approx 0.72\left[\frac{M_{p}-M_{s}}{0.5 \mathrm{M}_{\odot}}\right]\left[\frac{M}{1 \mathrm{M}_{\odot}}\right]^{-1 / 2}\left[\frac{a_{\text {bin }}}{0.2 \mathrm{AU}}\right]\left[\frac{e_{\text {bin }}}{0.2}\right]\left[\frac{a}{1 \mathrm{AU}}\right]^{-3 / 2} \mathrm{~km} / \mathrm{s} .
\end{aligned}
$$

Comparing this value with the disruption speed, it is clear that interpreting $v_{\text {force }}$ as a random motion leads to the simple prediction of destructive collisions for a broad range of particle sizes. Yet $v_{\text {force }}$ is the speed of a reference frame tied to a most circular orbit. Particles traveling on most circular orbits have no relative radial velocity; planetesimals may perturb each other and collide, but at velocities much smaller than $v_{\text {dest }}$ and $v_{\text {force }}$, promoting mergers instead of destruction.

This picture differs from the approach often taken in studies of circumbinary planet formation. For example, in Moriwaki \& Nakagawa (2004), Meschiari (2012), Paardekooper et al. (2012) and Lines et al. (2014), particles in $n$-body simulations are initialized on circular Keplerian orbits about the binary center of mass. Gas, if present, is assumed to have fluid elements on exactly circular sub-Keplerian orbits. From \&2 a particle trajectory with total eccentricity of $e=0$ is identical to a trajectory with equal parts free and forced eccentricity $\left(e_{\text {free }}=e_{\text {force }}\right)$, where the phase of the free part is chosen to yield an initial net eccentricity of zero $\left(\psi_{e}=\pi\right)$. The difficulty with these initial conditions is that the relative velocities are set with $v \sim e_{\text {free }} v_{\mathrm{K}} \sim v_{\text {force. }}$. It is then just a matter of time before precession of the free epicyclic motion drifts from the force motion, and particle orbits can cross. That time is

$$
T_{\text {pre }}=\frac{2 \pi}{\dot{\varpi}} \approx 67\left[\frac{a_{\text {bin }}}{0.2 \mathrm{AU}}\right]^{-2}\left[\frac{a}{1 \mathrm{AU}}\right]^{7 / 2}\left[\frac{M}{1 \mathrm{M}_{\odot}}\right]^{1 / 2}\left[\frac{\mu}{0.5 \mathrm{M}_{\odot}}\right]^{-1} \mathrm{yr},
$$

where the reduced mass is $\mu<0.5 M$. Entrainment of solids by the gas on sub-Keplerian orbits can reduce relative velocities, but only for particles of similar size (e.g., Marzari \& Scholl 2000). Thus, setting up protoplanetary disks with all objects having comparable free and forced eccentricities quickly dooms them to destruction.

Sometimes, the initial orbits of circumbinary planetesimals have no impact on the outcome of planet formation calculations. For example, if the free eccentricity is much larger than the forced eccentricity, then the binary's time varying potential is an unnoticed perturbation. This situation applies in the late stages of planet formation, when most of the mass is 
concentrated into a small number of large objects. At this stage, large objects have random velocities comparable to the escape velocity of the largest object (e.g., Goldreich et al. 2004; Kenyon \& Bromley 2006). Setting $v_{\text {esc }} \gtrsim v_{\text {force }}$, we obtain

$$
r_{\text {pro }} \gtrsim 1000\left[\frac{\rho}{3 \mathrm{~g} / \mathrm{cm}^{3}}\right]^{-1 / 2}\left[\frac{M}{1 \mathrm{M}_{\odot}}\right]^{1 / 2}\left[\frac{M_{p}-M_{s}}{0.5 \mathrm{M}_{\odot}}\right]\left[\frac{a_{\mathrm{bin}}}{0.2 \mathrm{AU}}\right]\left[\frac{e_{\mathrm{bin}}}{0.2}\right]\left[\frac{a}{1 \mathrm{AU}}\right]^{-3 / 2} \mathrm{~km} .
$$

Outside of the critical radius for stability, $a_{\text {crit }}$, a simulation with a collection of Pluto-size or larger objects should fail to see a difference between circumstellar and circumbinary environments. The simulations of Quintana \& Lissauer (2006) support this interpretation. Except for the effects of the destabilization of orbits near $a_{\text {crit }}$, ensembles of roughly lunar mass objects grow into a few terrestrial planets in circumstellar and circumbinary environments.

\subsection{Additional issues: resonances and binary evolution}

Once planetesimals have settled around most circular paths to grow by coagulation, the binary still influences their evolution. Overlapping resonances may stir particles and destabilize them (Wisdom 1980). However, these unstable orbits are typically close to the binary. For example, the 3:1 resonance, which corresponds to a singularity in the denominator of Equation (25), lies within 2.1 $a_{\text {bin }}$. As a guide, these unstable orbits are usually within the critical radius $a_{\text {crit }}$ identified by Holman \& Wiegert (1999). In some binary configurations instabilities extend beyond $a_{\text {crit }}$ (e.g., Musielak et al. 2005; Pichardo et al. 2005, 2008; Doolin \& Blundell 2011). Numerical simulations provide a straightforward way to identify these orbits (e.g. Popova \& Shevchenko 2013; Chavez et al. 2015) and to understand any impact on gas or solid particles in the planetary disk (e.g., Pierens \& Nelson 2008b).

Tidal evolution of the binary also complicates the long-term stability of circumbinary planetary systems. Changes in the orbital separation and eccentricity can change the positions of critical resonances, leading to instability in systems which had been stable. In the Pluto-Charon system, satellites become unstable when tidal evolution drives a relatively rapid expansion of the binary orbit (Ward \& Canup 2006; Lithwick \& Wu 2008; Cheng et al. 2014). For binaries composed of solar-type stars, tidal evolution on time scales of 1-100 Myr is important only for systems with orbital periods of 10 days or less (Meibom \& Mathieu 2005). Thus, tidal evolution of the central binary probably has little impact on the formation of most circumbinary planetary systems. 


\subsection{Summary}

Unless the collapse of a molecular cloud into a binary + disk system is significantly different from the collapse to a single star + disk system, we expect the stages of circumbinary planet formation to parallel those of circumstellar planet formation. As long as nonaxisymmetric structures such as spiral density waves are relatively unimportant, the time scales for gas damping, gas drag, collisional damping, and dynamical friction are all much shorter than typical precession times for the binary. Thus, material in a circumbinary disk should find its way onto most circular orbits around the binary in a similar way as material in a circumstellar disk damps onto circular orbits around a single star. Once material lies on or close to most circular orbits, small relative velocities of particles on adjacent orbits strongly favors growth over collisional disruption.

Precession of the inner binary has little impact on this conclusion. When a massive disk or a giant planet causes the inner binary to precess, the orbits of solid particles on most circular orbits maintain apsidal alignment. Because relevant damping time scales are short

$\left(\lesssim 10 \mathrm{yr}\right.$ for gas and $\lesssim 10^{3}$ yr for particles) compared with the binary precession time scales ( $\gtrsim 10^{4} \mathrm{yr}$ for precession induced by a massive disk, or longer with particle disks or gas giants beyond $1 \mathrm{AU}$ ) both gas and solids can maintain this apsidal alignment (see Equations (47) and (54) for damping times and Equations (50), (55) and (57) for binary precession periods). Most circular orbits remain the reference frame in which to measure relative velocities. Thus, planet formation proceeds in a standard fashion.

For calculations of circumbinary planet formation, starting with the right initial conditions for orbits of gaseous material or solid particles is crucial. Around a single star, it is sufficient to start material on orbits with modest eccentricity: precession is relatively unimportant and all damping processes lead to circular orbits. Around a binary, it is important to differentiate between the forced eccentricity driven by the binary and the free eccentricity relative to a most circular orbit around the binary (see also Pichardo et al. 2005, 2008). Starting particles on circular Keplerian orbits around the binary center of mass creates a free eccentricity relative to a most circular orbit. In general, this free eccentricity is comparable to the forced eccentricity induced by the binary. For eccentric binaries, this extra eccentricity produces spuriously large collision velocities which can lead to collisional destruction instead of growth by merger. To provide a proper evaluation of circumbinary planet formation, it is essential to begin with most circular orbits and then evaluate relative collision velocities for appropriate values of the free eccentricity. 


\section{Application: The Kepler circumbinary planets}

In the last decade, data from the Kepler satellite have started to paint a rich picture of circumbinary planetary systems (e.g., Armstrong et al. 2014, and references therein). The known systems (Dovle et al. 2011; Welsh et al. 2012; Orosz et al. 2012a.,b; Schwamb et al. 2013; Kostov et al. 2013, 2014) have fairly massive planets with radii $r \approx 0.25-0.76 R_{J}$ and semimajor axes $a \approx 0.3-1.1$ AU orbiting binaries with a broad range of mass ratios $(0.25-1)$ and eccentricities $(0.02-0.52)$. Although the number of circumbinary planetary systems is still rather small $(\sim 10)$, detection rates suggest these planets are roughly as common as planets around single stars (Armstrong et al. 2014).

At larger semimajor axes, circumbinary debris disks are also common. Among main sequence stars with FGK spectral types, single stars and binaries are equally likely to have debris disks (Trilling et al. 2007). In circumbinary debris disks, the disk and binary are often co-planar (e.g., Kennedy et al. 2012; Kennedy 2015). Small number statistics currently prevents robust conclusions, but it seems plausible that this structure is primordial: circumbinary disks form in the plane of the binary system (Kennedy et al. 2012; Kennedv 2015).

Understanding the structure of primordial circumbinary disks is also hampered by small sample sizes (e.g., Harris et al. 2012). Among multiple stars with ages of 1-3 Myr in the Taurus-Auriga molecular cloud, disks around single stars have masses similar to those of very wide binaries with $a \gtrsim 300 \mathrm{AU}\left(0.001-0.1 M_{\odot}\right.$, on the basis of dust mass estimates from millimeter fluxes; Andrews \& Williams 2005). Binaries with $a \approx 30-300$ AU (5-30 AU) have factor of $3-5(5-10)$ smaller disk masses. In three binaries with separations ranging from about $0.05 \mathrm{AU}$ to $10 \mathrm{AU}$, disk mass estimates yield $0.01-0.03 M_{\odot}$, comparable to the disks in single stars and wide binaries. For reference, a mass of $0.01 M_{\odot}$ corresponds to the Minimum-Mass Solar Nebula (MMSN), which is a the lower limit on the mass required to build the planets in the solar system (Weidenschilling 1977b; Havashi 1981).

Here, we survey known Kepler circumbinary systems to interpret how their orbital characteristics and size might inform us of their origin. While we reiterate some of the discussion in Leung \& Lee (2013), we also focus on how the observations might impact our understanding of planet formation scenarios, whether these planets formed in situ or migrated from some larger semimajor axis inward.

To frame the problem, we consider several different formation scenarios:

I. In situ formation with no migration. Planets grow by coagulation from nearby gas and dust. The final planetary mass is limited by the initial surface density of the proto- 
planetary disk. A Minimum-Mass Solar Nebula with $\Sigma_{0}=7 \mathrm{~g} / \mathrm{cm}^{2}$ in Equation (37) has $4 M_{\oplus}$ in solids inside of the snow line a $2.7 \mathrm{AU}$ and roughly $50 M_{\oplus}$ in solids inside $50 \mathrm{AU}$ (Weidenschilling 1977b; Hayashi 1981). The mass in gas is roughly $800 M_{\oplus}$ inside $2.7 \mathrm{AU}$ and roughly $3000 M_{\oplus}$ inside $50 \mathrm{AU}$. Numerical simulations suggest rocky planet formation is inefficient; collisional processes often lose significant amounts of mass during the assembly of Earth-mass and larger planets (e.g., Kenyon \& Bromley 2004, 2006; Ravmond et al. 2006, 2011). Augmenting the MMSN by a factor of 2-3 is sufficient to yield Earth-mass planets at $0.7-1$ AU in the solar system.

Producing a Neptune-mass planet in situ at $1 \mathrm{AU}$ is more challenging. Accreting sufficient gas from the disk to produce Neptune requires a $\sim 10 M_{\oplus}$ solid core (e.g., Pollack et al. 1996; Rafikov 2011; Rogers et al. 2011; Piso et al. 2015). In the standard MMSN, the mass in solids is insufficient to produce such a massive core. When the surface density of the disk is roughly 20 times the MMSN, coagulation models routinely yield $10 M_{\oplus}$ cores (e.g., Hansen \& Murray 2012; Schlichting 2014, see below).

In most numerical simulations, formation of 2-3 Earth mass or larger planets is common; producing a single planet is rare (e.g. Kenyon \& Bromley 2006; Raymond et al. 2011; Hansen \& Murray 2012; Hansen 2014). If these simulations are 'missing' an important piece of physics which allows several Earth-mass planets to merge into a single super-Earth mass planet, in situ formation of single super-Earth or Neptune mass planets might be possible in lower mass disks, e.g., 3-5 times the mass of the MMSN instead of $\sim 20$ times. Without a better understanding, we assume that formation of Neptune mass planets requires a very massive disk.

II. Migration then assembly. Precursor solid material is first moved from large $a$ to within $1 \mathrm{AU}$ of the host star. Planet formation then proceeds in situ (Hansen \& Murray 2012). In this way, the limited amount of solids available inside of $1 \mathrm{AU}$ is enhanced at early times; the delivery mechanism is uncertain. Because of the structure of the protoplanetary disk at small radii, gas accretion may be less efficient than beyond the snow line. Planets formed in this way may be "gas-starved" compared with more distant gas giants.

III. Migration through a gas disk. Gas giants form beyond the snow line, where the solid-togas ratio is a factor of $\sim 3$ larger (e.g., Kennedv \& Kenyon 2008). Planets move inward by exchanging torque with the gas disk (Ward 1997). Composition and structure of these planets are typical of the solar system's gas giants.

IV. Planet-planet scattering. Massive planets formed beyond the snow line gravitationally scatter one another, either inward toward the central mass or outward (Rasio \& Ford 1996; Chatterjee et al. 2008; Jurić \& Tremaine 2008; Raymond et al. 2010; Marzari et al. 
2010; Beaugé \& Nesvorný 2012; Moeckel \& Armitage 2012; Bromley \& Kenvon 2014). In a scattering event between two planets, if the more massive body is Neptune-size or larger (assuming typical solar system densities and orbital parameters for gas giants), then a broad range of outcomes is possible, including ejection from the system or the placement of a "hot Jupiter" near a single central star. High orbital eccentricity is the red flag for these scattering events.

For each of these modes, the initial mass of the disk and the epoch of planet formation are important considerations (e.g., Naiita \& Kenvon 2014, and references therein). In the Taurus-Auriga molecular cloud (e.g., Andrews \& Williams 2005; Andrews et al. 2013), the median mass in solids for disks around single stars drops from 50-100 $M_{\oplus}$ for protostars (ages of $0.1-0.5 \mathrm{Myr}$ ) to $10-20 M_{\oplus}$ for T Tauri stars (ages of 1-3 Myr). Observations of other star-forming regions suggest this evolution is typical (e.g., Andrews \& Williams 2007; Williams \& Cieza 2011). Thus, existence of Neptune-mass to Jupiter mass planets favors early formation in the massive disks of young protostars].

\subsection{Disk mass requirements for in situ growth}

For in situ formation, we estimate the surface density required to make a planet with mass $m$ and radius $r$. Each protoplanet accretes material from a "feeding zone" with radial width $\delta a$. Random motions of particles near the protoplanet set this width. When these particles have relative speeds smaller than the planet's escape velocity, the planet can accrete them. For particles with a free eccentricity, $e_{\text {free }} \lesssim v_{\text {esc }} / v_{\mathrm{K}}$ (e.g., Schlichting 2014). Thus,

$$
\Delta a \sim 2 a e_{\text {free }} \sim 2 a \frac{v_{\text {esc }}}{v_{\mathrm{K}}} \sim 2 a\left(\frac{2 a m}{r M}\right)^{1 / 2} .
$$

where $m$ and $r$ are the core's mass and radiu:5. By choosing a form for the surface density (we use $\Sigma \sim a^{-1.5}$ as in Equation (37)) and integrating over the feeding zone (excluding any unstable region inside of $a_{\text {crit }}$ ) we evaluate the minimum value for $\Sigma$ at the planet's position.

Figure 8 shows the results of applying this prescription to the Kepler circumbinary planets in Table 1. The minimum surface density required to build gas-accreting cores is

\footnotetext{
${ }^{4}$ For the solar system, radiometric analyses of meteorites similarly suggests formation of solids when the Sun had an age of 0.1-0.3 Myr (e.g., Kleine et al. 2009; Dauphas \& Chaussidon 2011).

${ }^{5}$ This result for the width of the feeding zone is somewhat larger and probably more realistic than the width derived for the more standard "isolation mass" (Greenzweig \& Lissauer 1990).
} 
roughly an order of magnitude more than the MMSN. Thus, any in situ formation model requires a massive disk. Models which form these planets well outside the snow line require much less massive disks.

This result allows us to eliminate in situ (no migration) models for all of the Kepler circumbinary planets. If the Kepler circumbinary planets are common (Armstrong et al. 2014), the precursor disks are also common. Among the youngests stars with ages of $0.1-0.5 \mathrm{Myr}$, however, disks with initial surface densities of 10-20 times the MMSN are exceedingly rare (Andrews \& Williams 2005). Because lower mass protostellar disks are common, the Kepler planets require a formation model with some form of migration or scattering.

\subsection{Resonances, instabilities and migration}

Although formation followed by radial migration is a plausible path for the Kepler circumbinary planets, unstable orbital resonances in the circumbinary environment pose clear obstacles to migration (Pierens \& Nelson 2007). Near certain commensurabilities, overlapping resonances excite large eccentricities (Wisdom 1982, 1983; Lecar et al. 2001). The Holman \& Wiegert (1999) condition that orbits are unstable if they are within a distance of $a_{\text {crit }}$ from the binary center of mass is a working guide; refinements are needed to identify the presence and "strength" of unstable resonances even outside of $a_{\text {crit }}$ (e.g., Popova \& Shevchenko 2013; Chavez et al. 2015).

Here, we assess whether the region around each binary in Table 1 is stable as a planet migrates inward. We consider two approaches: directly migrating the planet radially inward and, equivalently, expanding the binary. We show results for binary expansion models, which are more straightforward (and stringent) since they require adjusting only the Keplerian semimajor axis of the binary, instead of modifying the non-Keplerian orbit of the planet to mimic radial drift. Figure 9 shows the results for each Kepler planet at an equivalent radial drift rate of less than $10^{-5} \mathrm{AU} / \mathrm{yr}$ (typical for planets undergoing type I migration; Goldreich \& Tremaine 1980; Ward 1997; Tanaka et al. 2002) The particles in the Figure manage to get as close to their binary host as the planet's current position. Once inside, they become unstable near the 5:1 resonance, except for Kepler-34b, which becomes unstable near the 7:1 commensurability. Kepler-47b, orbiting the binary with the lowest eccentricity, is stable down to the 4:1 resonance. Thus, it seems plausible that the Kepler circumbinary planets, or smaller precursors (with fast damping times), could migrate through the potential minefield of resonances. Further tests would be needed to confirm whether the stability that we observe would remain with lower damping rates or slower migration times. 


\subsection{The Kepler circumbinary planets}

With constraints on in situ formation and migration from large distances established, we consider whether any of the four modes of planet formation is consistent with the bulk properties and orbit of each Kepler circumbinary planet listed in Table 1. We start with the first and most famous discovery, Kepler-16b.

- Kepler-16. The central binary, with a K-dwarf primary and an M-dwarf secondary, has significant eccentricity $\left(e_{\mathrm{bin}} \approx 0.16\right)$. The planet is a gas giant with radius of $0.7 R_{J}$ and a mass of $0.3 M_{J}$, orbiting at $0.7 \mathrm{AU}$ with low eccentricity, $\sim 0.01$ (Doyle et al. 2011). From the analytical theory (\$2), Leung \& Lee (2013) estimate that free and forced eccentricities are comparable, $e_{\text {free }} \approx e_{\text {force }} \approx 0.03$.

The high mass and low eccentricity of the planet favor the migrate-then-assemble and the migrate-in-gas modes of planet formation. In situ formation with no migration, although plausible, requires a very massive disk which is very uncommon among low mass stars with ages of 0.1-0.5 Myr (see Figure 8). Planet-planet scattering is less likely to result in this low-eccentricity configuration (e.g., Marzari et al. 2010; Beaugé \& Nesvorný 2012).

- Kepler-34. The binary has high eccentricity $\left(e_{\text {bin }} \approx 0.5\right)$ but the mass difference between the partners is small, within a few percent. Thus, the forced eccentricity at Kepler-34b's location is low, around 0.002. In contrast, the eccentricity of the planet is much larger, exceeding 0.2. The planet is about the same size as Kepler-16b and may be a Saturn analog. The high eccentricity and high mass favor a scattering scenario.

- Kepler-35. This binary has stars that are also nearly equal in mass. The planet, Kepler-35b, is nearly the same size as Kepler-16b and Kepler-34b, but has a much smaller eccentricity (0.048). As with Kepler-16b, the migrate-then-assemble or migratein-gas modes are strongly favored over the scattering or in situ with no migration formation modes.

- Kepler-38. The mass ratio of the binary is almost five to one; the eccentricity is around 0.1 . The planet is comparatively small, with a radius of $0.4 R_{J}$, suggesting a mass of roughly $20 M_{\oplus}$. Despite its low eccentricity (consistent with zero) and low mass, in situ formation with no migration is still problematic. Kepler-38b requires a surface density that is more than a factor of twenty larger than the MMSN. The low eccentricity similarly eliminates the scattering mode. Thus, we favor either the migrate-then-assemble or migrate-in-gas mode. 
- Kepler-47. The binary consists of a Sun-like star and a red dwarf on a nearly circular orbit. The forced eccentricity of its planet is low; the eccentricity of the planet is consistent with zero. With a radius just under $3 \mathrm{R}_{\oplus}$, Kepler-47b probably has the smallest mass of all the known circumbinary planets, perhaps $10 M_{\oplus}$. This planet has a companion about twice its radius, Kepler-47c, at an orbital distance of about $1 \mathrm{AU}$. The combined mass of the two planets works against in situ formation with no migration. Two planets orbiting inside the snow line requires a complicated scattering scenario (see Moeckel \& Armitage 2012). The migrate-then-assemble and the migratein-gas modes are less complicated. Improved statistics for this kind of circumbinary planetary system would provide better constraints on the scattering mode.

- PH1/Kepler-64. This binary has both a significant mass difference between its partners and a modestly high eccentricity (0.2). The resulting forced eccentricity is the largest of this sample (0.044). The planet PH1b is half the size of Jupiter, with an eccentricity of 0.05 ; thus, the free eccentricity is probably low. As with the other Kepler circumbinary planets, the large mass of the planet precludes in situ formation with no migration; the small orbital eccentricity eliminates most scattering models. Early formation in a massive disk allows either the migrate-then-assemble or the migrate-ingas models.

- Kepler-413. This low-eccentricity binary hosts a modest size planet, a third the radius of Jupiter. Like Kepler-38b, this mass is uncomfortably high for in situ formation without migration. Unlike Kepler-38b, Kepler-413b has a significant free eccentricity of more than 0.1. Large eccentricity tends to preclude the migrate-in-gas mode. However, either the migrate-then-assemble mode or a scattering model can produce a planet similar to Kepler-413b.

\subsection{Summary}

The circumbinary planets observed by Kepler are Neptune-size or bigger and located just beyond the critical radius around their host stars. Although in situ planet formation with no migration is a promising way to grow Earth mass planets at these distances, it is very unlikely to be responsible for the known Kepler circumbinary planets (Figure 8). The central issue - not enough mass to build Neptune-size planets - is circumvented by importing solids from beyond the snow line. Other formation mechanisms accomplish this mass transfer by invoking an inward radial flux of small particles within the disk, migrating fully-formed gas giants through the disk, or scattering gas giants from outside the snow-line where other large planets form. 
Migration seems to be involved in most of the Kepler planets. However, without larger samples of planets, it is impossible to distinguish between models where migration precedes assembly from those where migration follows assembly. All of the planets are too massive to allow in situ formation with no migration. However, the high free eccentricity observed in Kepler-34b and Kepler-413b are consistent with scattering events. Improved constraints on the orbits and bulk properties (mass, composition, spin, etc.) might allow more rigorous conclusions on their origin.

Here our most important contribution to the discussion of close-in planets (e.g., Schlichting 2014) is not to discriminate between formation mechanisms, but to emphasize that all are viable in the circumbinary environment at $a \gtrsim a_{\text {crit }}$. Thus, all issues concerning the formation of Neptunes and Jupiters inside the snow line for a single star carry over to the circumbinary case.

\section{Discussion}

Our main conclusion is that outside of a small region near a binary star, planet formation proceeds in much the same way as around a single star. This result stems from the existence of a family of "most circular" orbits around binaries that do not intersect, analogous to concentric circles around a single star. Gas, dust, and growing planets orbitally damp to these streamlined paths to avoid mutual collisions. The growth of planets, involving mergers, fragmentation, stirring and dynamical friction, takes place in the reference frame of guiding paths on these most circular orbits, just as it does in circumstellar disks. Without such paths, planetesimal orbits would inevitably mix at high velocity, leading to destruction, not growth.

These most circular paths are rooted in analytical theory. Lee \& Peale (2006) and Leung \& Lee (2013) lay out the foundation, describing how circumbinary orbits are approximated as linear combinations of (i) rapid, forced oscillations in response to the time-varying potential, (ii) slower, epicyclic motion - the "forced eccentricity" — that responds to the binary's eccentricity, and (iii) "free eccentricity" and inclination relative to the plane of the binary. The periapse of the forced eccentric orbit is aligned with the periapse of the binary. The free eccentricity and inclination have the same meaning for a circumbinary orbit as in a Keplerian system. There is precession of the free eccentric orbit in the circumbinary case, for the same reason as in the case of a single, oblate star (quadrupole and higher order contributions to the time-averaged gravitational potential).

We place this analytical framework in the context of planet formation. Our results 
demonstrate that (i) most circular orbits exist for binaries even when their eccentricity is large enough that the theory - based on a perturbative approach — becomes questionable; (ii) non-intersecting, most circular orbits exist in the presence of a massive disk and a large planet; (iii) these orbits remain apsidally aligned with the binary - if the binary precesses, the forced epicyclic paths in the disk also precess; and (iv) the response of a satellite to external perturbations (e.g., stirring from a distant planet) is the same as it would be around a single star, in the reference frame of a circular guiding center. Thus, planets can form in situ as close as a few times the orbital separation of their binary hosts. Nearer to home, satellites of the Pluto-Charon binary may have formed in a similar way (Kenyon \& Bromley 2014).

Extending these ideas to include gas within a protoplanetary disk, disk gravity and gas pressure slightly distort the shape of most circular orbits but not their alignment with the binary. Although deviations from strictly circular geometry introduce new hydrodynamical effects (e.g., Ogilvie \& Barker 2014; Barker \& Ogilvie 2014), circumbinary gas streamlines plausibly settle on these most circular paths as they do on circular orbits around a point mass. Interactions with solid particles are also similar. The "headwind" felt by planetesimals traveling through the gas is nearly the same as in a circumstellar disk (Figure 5). As the gas dissipates in time, streamlines and planetesimals damp to the same set of most circular orbits.

To explore whether our results are sensitive to instabilities of circumbinary orbits, we also consider resonant excitations (Artymowicz \& Lubow 1994; Kley \& Haghighipour 2014). In our tests, the Kepler circumbinary planets are all beyond the outermost unstable resonance around their host. For most systems, this resonance is the 5:1 commensurability, close to the prediction of Holman \& Wiegert (1999). We define stability over a limited time frame $\left(10^{3}-10^{5} \mathrm{yr}\right)$, not the age of the planetary systems. Nonetheless, our simulations suggest that with typical damping rates (Weidenschilling 1977b; Goldreich et al. 2004; Chiang \& Youdin 2010) and migration times (Ward 1997), both in situ formation and migration of planets or their precursors are plausible.

Issues that we do not address include possible non-axisymmetric structure in a massive circumbinary disk (Pelupessy \& Portegies Zwart 2013), or the physics near the edge of the stability zone at a distance of a few times the binary separation. Simulations of gas and particle disks can shed light on whether material is pushed outward by torque exchange with the binary or lost in the unstable zone (Artymowicz \& Lubow 1996; Günther \& Klev 2002). Furthermore, we do not consider tidal evolution of the binary (e.g., Hurlev et al. 2002). Depending on the nature of the evolution, variations in binary separation and eccentricity will affect the stability of orbits in circumbinary planetary system. For an intriguing example, 
see the discussion by Ward \& Canup (2006) on resonant transport of moons in orbits around the Pluto-Charon binary as it tidally expands (Bromley \& Kenyon 2015).

Our results stand in contrast to previous theoretical studies of circumbinary planet formation. The prevailing view is that dynamical excitation of planetesimals by the binary leads to destructive collisions (Moriwaki \& Nakagawa 2004; Meschiari 2012; Paardekooper et al. 2012; Rafikov 2013; Xie 2013; Lines et al. 2014). In this interpretation, if the binary's eccentricity is even modestly high, $e_{\text {bin }} \approx 0.2$, epicyclic velocities eventually exceed the shattering speed for all but the largest planetesimals over a significant range of orbital distances. Entrainment in a gas disk (e.g., Marzari \& Scholl 2000), or precession from disk gravity (Rafikov 2013; Silsbee \& Rafikov 2015a) mitigate the situation only for some range of planetesimal sizes or distant regions of a protoplanetary disk. In this view, planet formation close to the binary is impossible.

These conclusions depend on the assumption that gas and planetesimals are initially on orbits with no eccentricity in the Keplerian sense (see Figure 1 in Lines et al. 2014). In light of analytical theory $(\S 2)$, this choice endows particles with equal amounts of free and forced eccentricity. As the free part drifts in phase, collisions destroy growing protoplanets. A more realistic approach to modeling an unstirred disk is to set the gas and planetesimals on most circular orbits with no free eccentricity. Absent any stirring, planetesimals in a particle disk orbit an eccentric binary host indefinitely without colliding. If planetesimals develop small $e$ and $i$ about the most circular orbits, collision velocities are modest, just as for planetesimals with modest $e$ and $i$ about a circular orbit around a single star. Modest collision velocities promote growth instead of destruction. Planet formation close to the binary is then robust.

Finally, we review characteristics of Kepler circumbinary planets in light of our results. If circumbinary planets form roughly in situ, the orbital characteristics and sizes of these planets require very large initial surface densities (10-20 times the MMSN). Although these high surface densities appear to preclude in situ models with no migration, the migratethen-assemble picture of Hansen \& Murrav (2012) is viable. As a plausible alternative, migrate-in-gas models allow these planets to form beyond the snow line and migrate inward through the gas. For this set of Kepler planets, migration through gas avoids unstable resonances around the binary. For Kepler-34b and Kepler-413b, formation beyond the snow line followed by a scattering event is also a reasonable scenario.

In general, beyond the inner unstable cavity around a stellar binary, the evolution of solids on most circular orbits differs little from the evolution of solids on circular orbits around a single star. Thus, the standard issues of formation around single stars (e.g., planetesimal formation, migration, resonances, scattering, etc) have clear parallels in circumbinary disks (for a summary of the issues in single stars, see Schlichting 2014). 
We conclude with predictions for circumbinary planetary systems. In our scenario, planets are as prevalent around binaries as around single stars. Furthermore, relative to coplanar, most circular orbits, these planets should have the same distribution of orbital elements (free eccentricity and inclination) as their circumstellar cousins. Data from the full Kepler catalog indeed suggest that the planets have comparable rates around binaries and single stars (Armstrong et al. 2014). Tatooine sunsets may be common after all.

We thank the referee for a timely and thoughtful report that helped is to hone our presentation. We also thank N. Georgakarakos, J. Pringle, R. Rafikov, P. Thébault and A. Youdin for helpful comments on the manuscript. We gratefully acknowledge NASA for support through the Astrophysics Theory and Origins of Solar Systems programs (grant NNX10AF35G) and through the Outer Planets Program (grant NNX11AM37G). We also acknowledge NASA for a generous allocation of time on the 'discover' supercomputing cluster. This research has made use of the Exoplanet Orbit Database and the Exoplanet Data Explorer at exoplanets.org.

\section{REFERENCES}

Adachi, I., Hayashi, C., \& Nakazawa, K. 1976, Progress of Theoretical Physics, 56, 1756

Andrews, S. M., Rosenfeld, K. A., Kraus, A. L., \& Wilner, D. J. 2013, ApJ, 771, 129

Andrews, S. M., \& Williams, J. P. 2005, ApJ, 631, 1134

-. 2007, ApJ, 671, 1800

Armstrong, D. J., Osborn, H. P., Brown, D. J. A., et al. 2014, MNRAS, 444, 1873

Artymowicz, P., \& Lubow, S. H. 1994, ApJ, 421, 651

-. 1996, ApJ, 467, L77

Barker, A. J., \& Ogilvie, G. I. 2014, MNRAS, 445, 2637

Beaugé, C., \& Nesvorný, D. 2012, ApJ, 751, 119

Benz, W., \& Asphaug, E. 1999, Icarus, 142, 5

Beuermann, K., Buhlmann, J., Diese, J., et al. 2011, A\&A, 526, A53

Birnstiel, T., Andrews, S. M., \& Ercolano, B. 2012, A\&A, 544, A79 
Birnstiel, T., Dullemond, C. P., \& Brauer, F. 2010, A\&A, 513, A79+

Borucki, W. J., Koch, D. G., Basri, G., et al. 2011, ApJ, 736, 19

Brauer, F., Dullemond, C. P., \& Henning, T. 2008, A\&A, 480, 859

Bromley, B. C., \& Kenyon, S. J. 2006, AJ, 131, 2737

—. 2011a, ApJ, 731, 101

—. 2011b, ApJ, 735, 29

—. 2014, ApJ, 796, 141

—. 2015, ArXiv e-prints, arXiv:1503.06805

Burke, C. J., Bryson, S. T., Mullally, F., et al. 2014, ApJS, 210, 19

Cassan, A., Kubas, D., Beaulieu, J.-P., et al. 2012, Nature, 481, 167

Cassen, P., \& Moosman, A. 1981, Icarus, 48, 353

Chambers, J. E., \& Wetherill, G. W. 1998, Icarus, 136, 304

Chatterjee, S., Ford, E. B., Matsumura, S., \& Rasio, F. A. 2008, ApJ, 686, 580

Chavez, C. E., Georgakarakos, N., Prodan, S., et al. 2015, MNRAS, 446, 1283

Cheng, W. H., Peale, S. J., \& Lee, M. H. 2014, Icarus, 241, 180

Chiang, E., \& Youdin, A. N. 2010, Annual Review of Earth and Planetary Sciences, 38, 493

Chiang, E. I., \& Goldreich, P. 1997, ApJ, 490, 368

Chirikov, B. V. 1959, Soviet Physics Doklady, 4, 390

—. 1979, Phys. Rep., 52, 263

Cumming, A., Butler, R. P., Marcy, G. W., et al. 2008, PASP, 120, 531

Dauphas, N., \& Chaussidon, M. 2011, Annual Review of Earth and Planetary Sciences, 39, 351

Davis, D. R., Chapman, C. R., Weidenschilling, S. J., \& Greenberg, R. 1985, Icarus, 63, 30

Dent, W. R. F., Thi, W. F., Kamp, I., et al. 2013, PASP, 125, 477 
Dong, S., \& Zhu, Z. 2013, ApJ, 778, 53

Doolin, S., \& Blundell, K. M. 2011, MNRAS, 418, 2656

Doyle, L. R., Carter, J. A., Fabrycky, D. C., et al. 2011, Science, 333, 1602

Garaud, P., Meru, F., Galvagni, M., \& Olczak, C. 2013, ApJ, 764, 146

Georgakarakos, N., \& Eggl, S. 2015, ApJ, 802, 94

Goldreich, P., Lithwick, Y., \& Sari, R. 2004, ARA\&A, 42, 549

Goldreich, P., \& Tremaine, S. 1980, ApJ, 241, 425

Goldreich, P., \& Tremaine, S. D. 1978, Icarus, 34, 227

Gould, A., Dong, S., Gaudi, B. S., et al. 2010, ApJ, 720, 1073

Greenberg, R., Hartmann, W. K., Chapman, C. R., \& Wacker, J. F. 1978, Icarus, 35, 1

Greenzweig, Y., \& Lissauer, J. J. 1990, Icarus, 87, 40

Günther, R., \& Kley, W. 2002, A\&A, 387, 550

Han, E., Wang, S. X., Wright, J. T., et al. 2014, PASP, 126, 827

Hansen, B. M. S. 2014, ArXiv e-prints, arXiv:1403.6553

Hansen, B. M. S., \& Murray, N. 2012, ApJ, 751, 158

Harris, R. J., Andrews, S. M., Wilner, D. J., \& Kraus, A. L. 2012, ApJ, 751, 115

Hayashi, C. 1981, Progress of Theoretical Physics Supplement, 70, 35

Heppenheimer, T. A. 1978, A\&A, 65, 421

Holman, M. J., \& Wiegert, P. A. 1999, AJ, 117, 621

Hornung, P., Pellat, R., \& Barge, P. 1985, Icarus, 64, 295

Housen, K. R., \& Holsapple, K. A. 1999, Icarus, 142, 21

Howard, A. W., Marcy, G. W., Johnson, J. A., et al. 2010, Science, 330, 653

Howard, A. W., Marcy, G. W., Bryson, S. T., et al. 2012, ApJS, 201, 15

Hurley, J. R., Tout, C. A., \& Pols, O. R. 2002, MNRAS, 329, 897 
Jurić, M., \& Tremaine, S. 2008, ApJ, 686, 603

Kennedy, G. M. 2015, MNRAS, 447, L75

Kennedy, G. M., \& Kenyon, S. J. 2008, ApJ, 673, 502

Kennedy, G. M., Wyatt, M. C., Sibthorpe, B., et al. 2012, MNRAS, 426, 2115

Kenyon, S. J., \& Bromley, B. C. 2001, AJ, 121, 538

-. 2002, AJ, 123, 1757

-. 2004, ApJ, 602, L133

-. 2005, AJ, 130, 269

—. 2006, AJ, 131, 1837

—. 2008, ApJS, 179, 451

-. 2010, ApJS, 188, 242

-. 2014, AJ, 147, 8

—. 2015, ArXiv e-prints, arXiv:1501.05659

Kenyon, S. J., \& Luu, J. X. 1998, AJ, 115, 2136

Kleine, T., Touboul, M., Bourdon, B., et al. 2009, Geochim. Cosmochim. Acta, 73, 5150

Kley, W., \& Haghighipour, N. 2014, A\&A, 564, A72

Kostov, V. B., McCullough, P. R., Hinse, T. C., et al. 2013, ApJ, 770, 52

Kostov, V. B., McCullough, P. R., Carter, J. A., et al. 2014, ApJ, 784, 14

Laibe, G., Gonzalez, J.-F., \& Maddison, S. T. 2012, A\&A, 537, A61

Lecar, M., Franklin, F. A., Holman, M. J., \& Murray, N. J. 2001, ARA\&A, 39, 581

Lee, M. H., \& Peale, S. J. 2006, Icarus, 184, 573

Leung, G. C. K., \& Lee, M. H. 2013, ApJ, 763, 107

Lightman, A. P. 1974, ApJ, 194, 419

Lin, D. N. C., \& Papaloizou, J. 1979, MNRAS, 188, 191 
Lin, D. N. C., \& Pringle, J. E. 1976, in IAU Symposium, Vol. 73, Structure and Evolution of Close Binary Systems, ed. P. Eggleton, S. Mitton, \& J. Whelan, 237

Lines, S., Leinhardt, Z. M., Paardekooper, S., Baruteau, C., \& Thebault, P. 2014, ApJ, 782, L11

Lissauer, J. J. 1987, Icarus, 69, 249

Lithwick, Y., \& Wu, Y. 2008, ArXiv e-prints, arXiv:0802.2939

Lynden-Bell, D., \& Pringle, J. E. 1974, MNRAS, 168, 603

Macintosh, B., Graham, J. R., Ingraham, P., et al. 2014, Proceedings of the National Academy of Science, 111, 12661

Marzari, F., Baruteau, C., \& Scholl, H. 2010, A\&A, 514, L4

Marzari, F., \& Scholl, H. 2000, ApJ, 543, 328

Mayor, M., Lovis, C., \& Santos, N. C. 2014, Nature, 513, 328

Mayor, M., Marmier, M., Lovis, C., et al. 2011, ArXiv e-prints, arXiv:1109.2497

Meibom, S., \& Mathieu, R. D. 2005, ApJ, 620, 970

Meschiari, S. 2012, ApJ, 752, 71

-. 2014, ApJ, 790, 41

Moeckel, N., \& Armitage, P. J. 2012, MNRAS, 419, 366

Moriwaki, K., \& Nakagawa, Y. 2004, ApJ, 609, 1065

Mullally, F., Coughlin, J. L., Thompson, S. E., et al. 2015, ApJS, 217, 31

Murray, C. D., \& Dermott, S. F. 1999, Solar system dynamics (Princeton: Princeton University Press)

Musielak, Z. E., Cuntz, M., Marshall, E. A., \& Stuit, T. D. 2005, A\&A, 434, 355

Najita, J. R., \& Kenyon, S. J. 2014, MNRAS, 445, 3315

Ogilvie, G. I. 2001, MNRAS, 325, 231

Ogilvie, G. I., \& Barker, A. J. 2014, MNRAS, 445, 2621 
Ohtsuki, K. 1999, Icarus, 137, 152

Ohtsuki, K., Stewart, G. R., \& Ida, S. 2002, Icarus, 155, 436

Orosz, J. A., Welsh, W. F., Carter, J. A., et al. 2012a, Science, 337, 1511

-. 2012b, ApJ, 758, 87

Paardekooper, S.-J., Leinhardt, Z. M., Thébault, P., \& Baruteau, C. 2012, ApJ, 754, L16

Pelupessy, F. I., \& Portegies Zwart, S. 2013, MNRAS, 429, 895

Pichardo, B., Sparke, L. S., \& Aguilar, L. A. 2005, MNRAS, 359, 521

-. 2008, MNRAS, 391, 815

Pierens, A., \& Nelson, R. P. 2007, A\&A, 472, 993

-. 2008a, A\&A, 478, 939

-. 2008b, A\&A, 483, 633

Pinte, C., \& Laibe, G. 2014, A\&A, 565, A129

Piso, A.-M. A., Youdin, A. N., \& Murray-Clay, R. A. 2015, ApJ, 800, 82

Pollack, J. B., Hubickyj, O., Bodenheimer, P., et al. 1996, Icarus, 124, 62

Popova, E. A., \& Shevchenko, I. I. 2013, ApJ, 769, 152

Pringle, J. E. 1981, ARA\&A, 19, 137

-. 1991, MNRAS, 248, 754

Quintana, E. V., \& Lissauer, J. J. 2006, Icarus, 185, 1

Rafikov, R. R. 2004, AJ, 128, 1348

-. 2011, ApJ, 727, 86

—. 2013, ApJ, 764, L16

Rasio, F. A., \& Ford, E. B. 1996, Science, 274, 954

Raymond, S. N., Armitage, P. J., \& Gorelick, N. 2010, ApJ, 711, 772

Raymond, S. N., Quinn, T., \& Lunine, J. I. 2006, Icarus, 183, 265 
Raymond, S. N., Armitage, P. J., Moro-Martín, A., et al. 2011, A\&A, 530, A62

Rogers, L. A., Bodenheimer, P., Lissauer, J. J., \& Seager, S. 2011, ApJ, 738, 59

Safronov, V. S. 1969, Evoliutsiia doplanetnogo oblaka. (Evolution of the Protoplanetary Cloud and Formation of the Earth and Planets, Nauka, Moscow [Translation 1972, NASA TT F-677] (1969.)

Schlichting, H. E. 2014, ApJ, 795, L15

Schneider, J., Dedieu, C., Le Sidaner, P., Savalle, R., \& Zolotukhin, I. 2011, A\&A, 532, A79

Scholl, H., Marzari, F., \& Thébault, P. 2007, MNRAS, 380, 1119

Schwamb, M. E., Orosz, J. A., Carter, J. A., et al. 2013, ApJ, 768, 127

Silsbee, K., \& Rafikov, R. R. 2015a, ArXiv e-prints, arXiv:1504.00460

-. 2015b, ApJ, 798, 71

Spaute, D., Weidenschilling, S. J., Davis, D. R., \& Marzari, F. 1991, Icarus, 92, 147

Stewart, G. R., \& Wetherill, G. W. 1988, Icarus, 74, 542

Syer, D., \& Clarke, C. J. 1992, MNRAS, 255, 92

Szebehely, V. 1967, Theory of orbits. The restricted problem of three bodies (Academic Press, New York, NY)

Tamura, M. 2014, in American Astronomical Society Meeting Abstracts, Vol. 224, American Astronomical Society Meeting Abstracts 224, 301.03

Tanaka, H., Takeuchi, T., \& Ward, W. R. 2002, ApJ, 565, 1257

Terebey, S., Shu, F. H., \& Cassen, P. 1984, ApJ, 286, 529

Thébault, P., Marzari, F., \& Scholl, H. 2006, Icarus, 183, 193

Trilling, D. E., Stansberry, J. A., Stapelfeldt, K. R., et al. 2007, ApJ, 658, 1289

Ward, W. R. 1981, Icarus, 47, 234

-. 1997, Icarus, 126, 261

Ward, W. R., \& Canup, R. M. 2006, Science, 313, 1107 
Weidenschilling, S. J. 1977a, MNRAS, 180, 57

—. 1977b, Ap\&SS, 51, 153

-. 1989, Icarus, 80, 179

Weidenschilling, S. J., \& Cuzzi, J. N. 1993, in Protostars and Planets III, ed. E. H. Levy \& J. I. Lunine (University of Arizona Press, Tucson, AZ), 1031-1060

Welsh, W. F., Orosz, J. A., Carter, J. A., et al. 2012, Nature, 481, 475

Wetherill, G. W. 1980, ARA\&A, 18, 77

Wetherill, G. W., \& Stewart, G. R. 1993, Icarus, 106, 190

Williams, J. P., \& Cieza, L. A. 2011, ARA\&A, 49, 67

Windmark, F., Birnstiel, T., Ormel, C. W., \& Dullemond, C. P. 2012, A\&A, 544, L16

Wisdom, J. 1980, AJ, 85, 1122

-. 1982, AJ, 87, 577

-. 1983, Icarus, 56, 51

Xie, J. W. 2013, Acta Astronomica Sinica, 54, 79

Yorke, H. W., Bodenheimer, P., \& Laughlin, G. 1993, ApJ, 411, 274

Yoshida, H. 1990, Physics Letters A, 150, 262

Youdin, A. N. 2011, ApJ, 742, 38

Youdin, A. N., \& Chiang, E. I. 2004, ApJ, 601, 1109

Youdin, A. N., \& Kenyon, S. J. 2013, in Planets, Stars and Stellar Systems. Volume 3: Solar and Stellar Planetary Systems, ed. T. D. Oswalt, L. M. French, \& P. Kalas (Dordrecht: Springer Science \& Business Media), 1

Youdin, A. N., Kratter, K. M., \& Kenyon, S. J. 2012, ApJ, 755, 17

Zechmeister, M., Kürster, M., Endl, M., et al. 2013, A\&A, 552, A78 
Table 1: Kepler binaries. Along with orbital parameters are estimates of the innermost stable orbit radius $\left(a_{\text {crit }}\right)$ and the forced eccentricity $\left(e_{\text {force }}\right)$.

\begin{tabular}{l|llcc|ccc|cc}
\hline \hline & $M_{p}\left(M_{\odot}\right)$ & $M_{s}\left(M_{\odot}\right)$ & $a_{\text {bin }}(\mathrm{AU})$ & $e_{\text {bin }}$ & $a(\mathrm{AU})$ & $e$ & $r\left(\mathrm{R}_{J}\right)$ & $a_{\text {crit }}(\mathrm{AU})$ & $e_{\text {force }}$ \\
\hline Kepler-16 $^{\mathrm{a}}$ & 0.687 & 0.202 & 0.224 & 0.160 & 0.720 & 0.024 & 0.75 & 0.646 & 0.034 \\
Kepler-34 $^{\mathrm{a}}$ & 1.049 & 1.022 & 0.228 & 0.521 & 1.086 & 0.209 & 0.76 & 0.833 & 0.002 \\
Kepler-35 $^{\mathrm{a}}$ & 0.885 & 0.808 & 0.176 & 0.142 & 0.605 & 0.048 & 0.73 & 0.496 & 0.002 \\
Kepler-38 $^{\mathrm{b}}$ & 0.949 & 0.249 & 0.147 & 0.103 & 0.464 & $<0.032$ & 0.39 & 0.389 & 0.024 \\
Kepler-47 $^{\mathrm{c}}$ & 1.043 & 0.362 & 0.084 & 0.023 & 0.296 & $<0.035$ & 0.27 & 0.203 & 0.004 \\
PH1 $^{\mathrm{d}}$ & 1.528 & 0.378 & 0.174 & 0.212 & 0.634 & 0.054 & 0.55 & 0.527 & 0.044 \\
Kepler-413 $^{\mathrm{e}}$ & 0.820 & 0.542 & 0.099 & 0.037 & 0.355 & 0.118 & 0.39 & 0.253 & 0.003
\end{tabular}

${ }^{a}$ See Dovle et al. (2011) and Welsh et al. (2012); orbital elements are from Leung \& Lee (2013, Table 1); Orosz et al. (2012b).

Orosz et al. (2012a); A second planet $\left(r=0.41 \mathrm{R}_{J}\right)$ is at $\sim 1 \mathrm{AU}$.

'Schwamb et al. (2013) and Kostov et al. (2013); Kepler-64.

Kostov et al. (2014). 


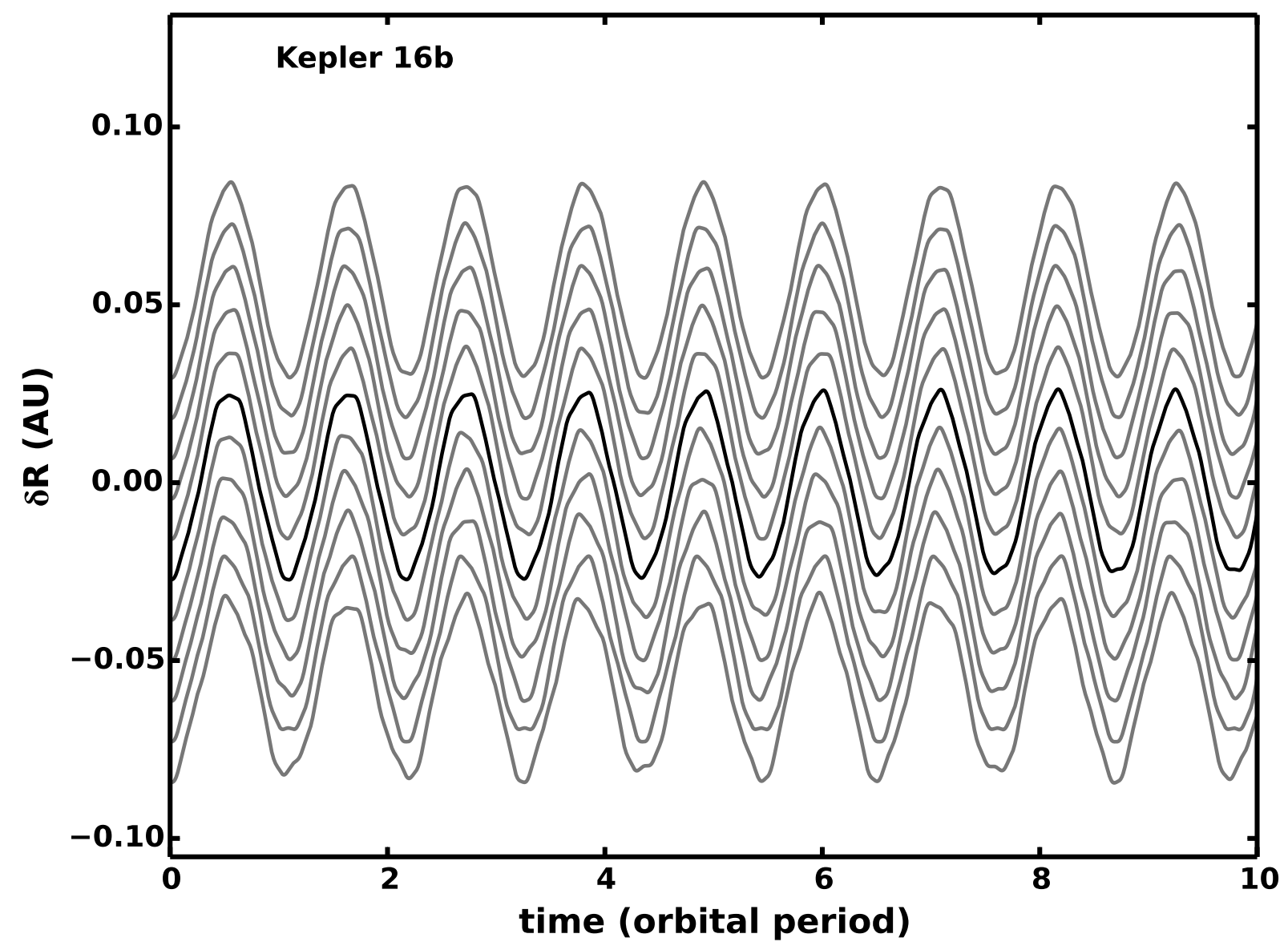

Fig. 1. - The radial excursion of Kepler-16b on most circular orbits. The dark curve shows the radial excursion of a satellite at the orbital position of the planet in the absence of free eccentricity and inclination, plotted as a function of orbital phase (in units of the planet's orbital period). The gray curves show orbits at slightly displaced orbital distances. The trajectories are dominated by the forced eccentric orbit. Comparatively small higherfrequency oscillations are visible in the curves. Despite the appearance that these oscillations are not in phase between the curves, the orbits do not cross. 


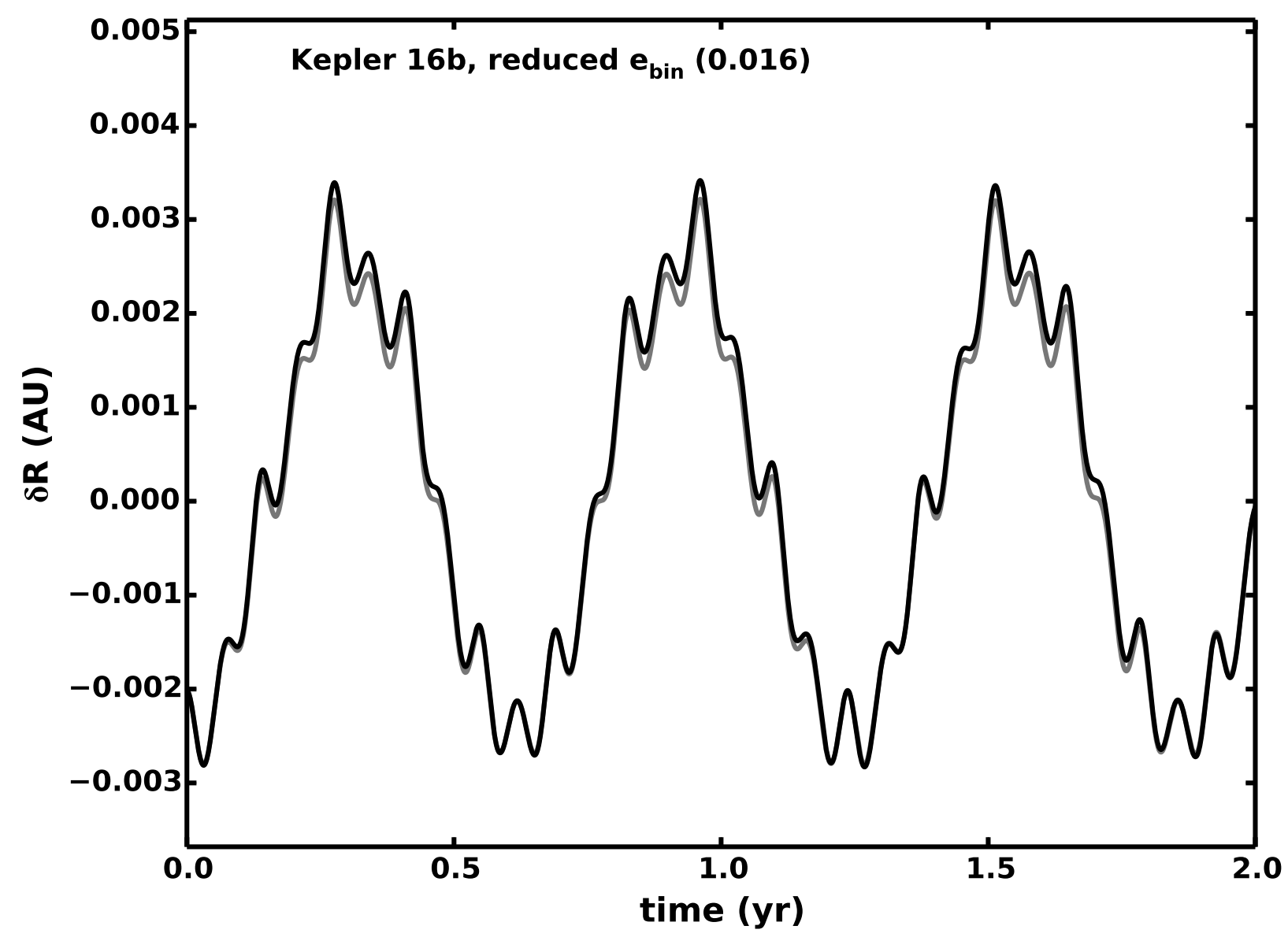

Fig. 2.- Illustration of radial excursions of a satellite on a most circular orbit. The orbital configuration and binary masses are derived from Kepler-16 and its planet Kepler-16b at $a=0.70 \mathrm{AU}$. The binary eccentricity has been reduced by a factor of 10 from the real system; the plot then distinguishes the forced eccentricity (larger amplitude, driven at the orbital period, $\sim 0.6 \mathrm{yr}$ ) and the high-frequency oscillations (smaller amplitude driven at the binary's orbital period and the synodic period of the satellite relative to the binary, $\sim 0.1 \mathrm{yr}$ ). The black curve is from simulation while the gray curve is from analytical theory (Equation (21)). 


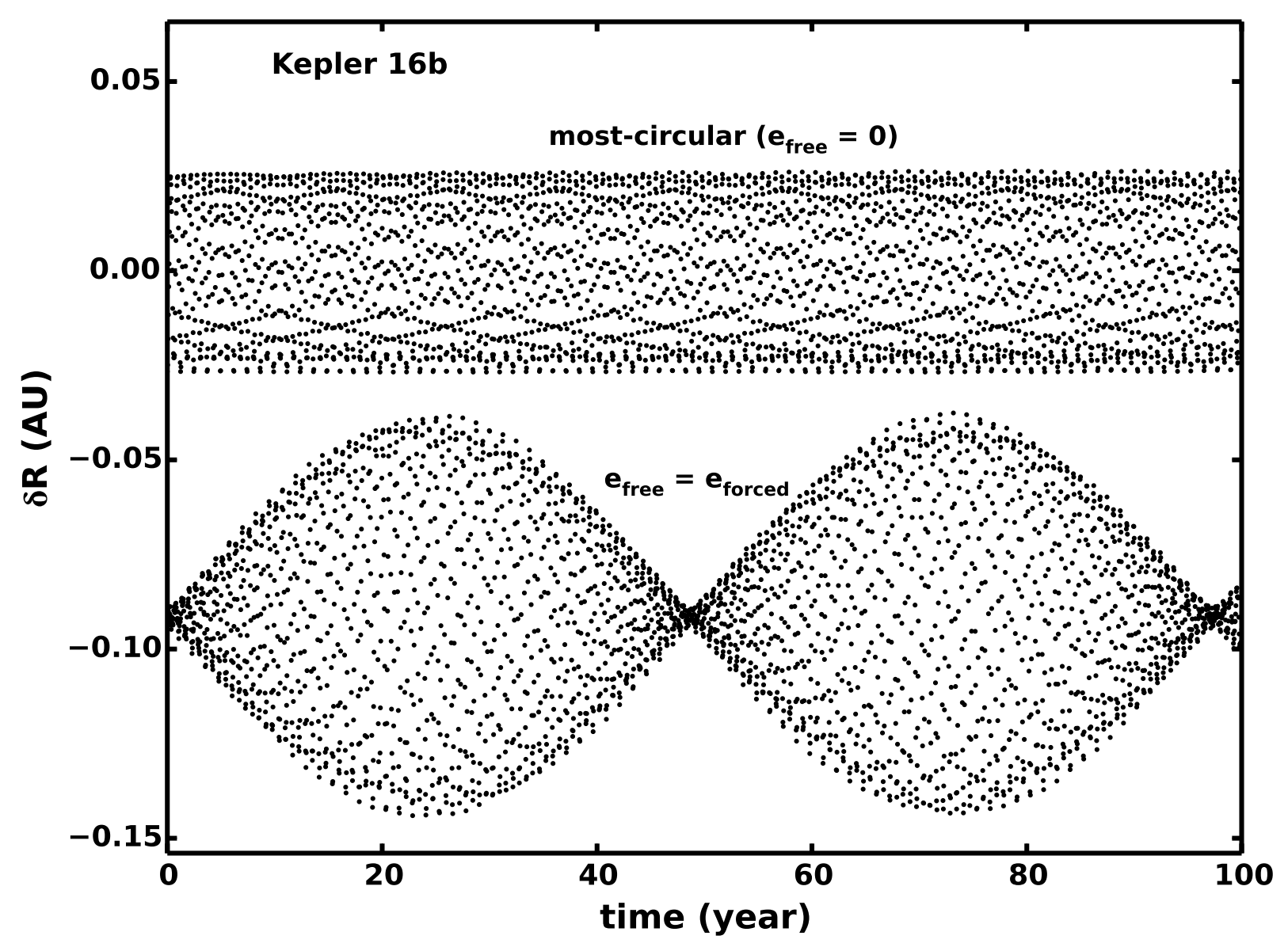

Fig. 3.- Comparison between a most circular orbit and one initially on the circular path of the guiding center (zero eccentricity). The most circular path (upper curve) is non-precessing, with excursions from the guiding center that have a maximum amplitude that does not drift over time. In contrast, a particle that is set up on a "circular" orbit - launching the particle from its guiding center with the speed of uniform circular motion about the binary center of mass - has a mixture of free and force eccentricities in equal measure. The relative phase allows zero eccentricity at the start. The result (lower curve, displaced from the upper one for clarity) is the beat pattern with a frequency given by the precession rate of the free eccentric orbit (Equation (33)). 


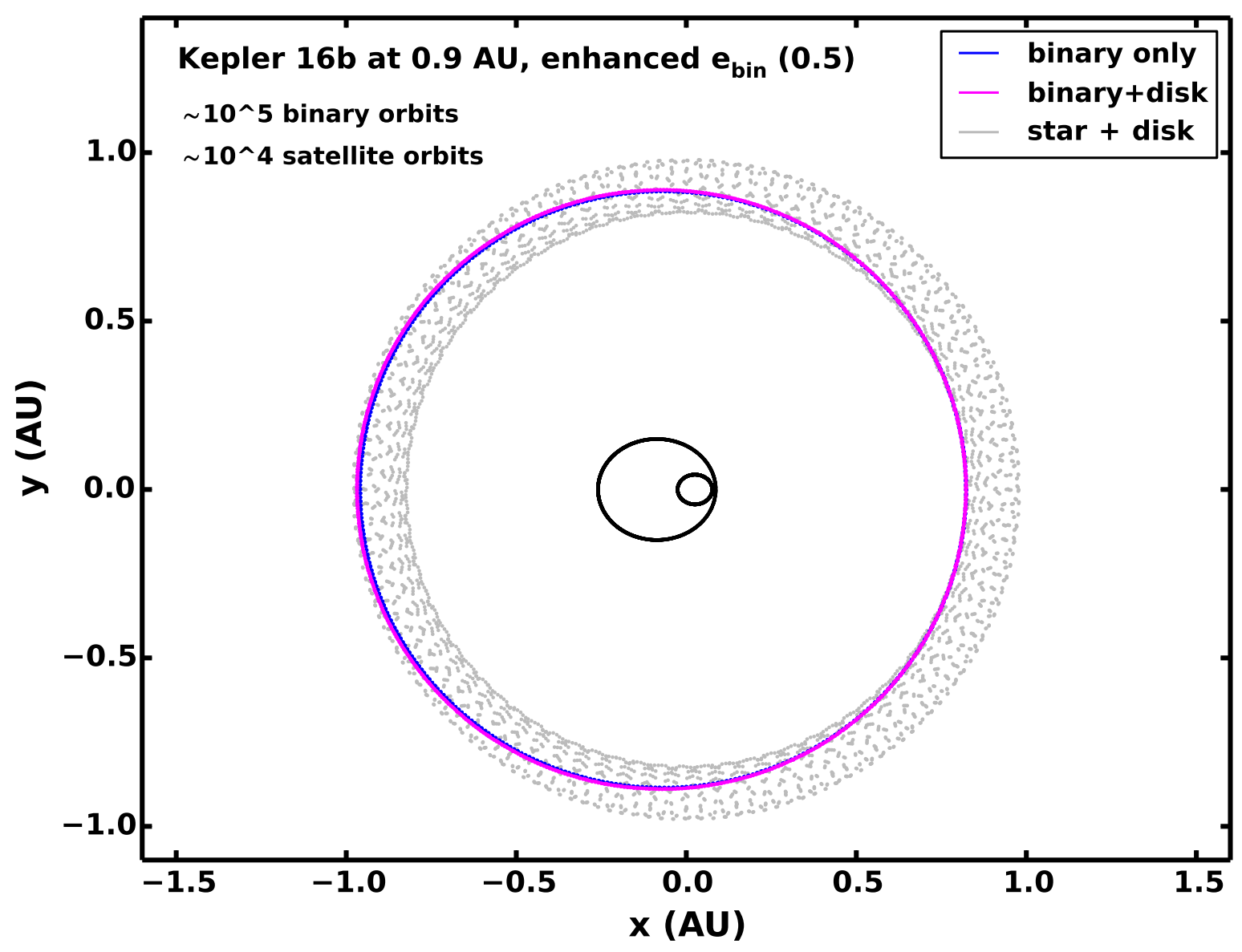

Fig. 4.- Simulated most circular orbits around a binary with moderately high eccentricity. The orbital configuration is derived from Kepler-16, as in the previous figures, but the binary eccentricity is set to 0.5 , and the satellite is placed at $0.9 \mathrm{AU}$, just beyond the critical radius for stability $\left(a_{\text {crit }}=0.86 \mathrm{AU}\right.$ ). The center of mass of the system is at the origin in this $x-y$ map of the plane of the binary. The secondary's pericenter is on the positive $x$-axis. The satellite (blue dots) tracks a narrow elliptical path with an eccentricity of $e_{\text {force }} \approx 0.08$, fixed and aligned with the binary for $\sim 10^{4}$ orbital periods $\left(\sim 10^{5}\right.$ binary orbits). When the potential of a massive disk ( $\Sigma$ of $2000 \mathrm{~g} / \mathrm{cm}^{2}$ at $1 \mathrm{AU}$ ) is included, the satellite's most circular path does not precess (magenta points, mixed in with the blue ones). For reference, we show samples of a satellite orbiting a single star with Kepler-16's total mass, also in this disk potential (gray points). In this case, the precession of the satellite's argument of periastron is rapid $(\sim 0.005 \mathrm{rad} / \mathrm{yr})$; the points fill an annular swath. In these simulations, there is no interaction between the disk and the binary. 


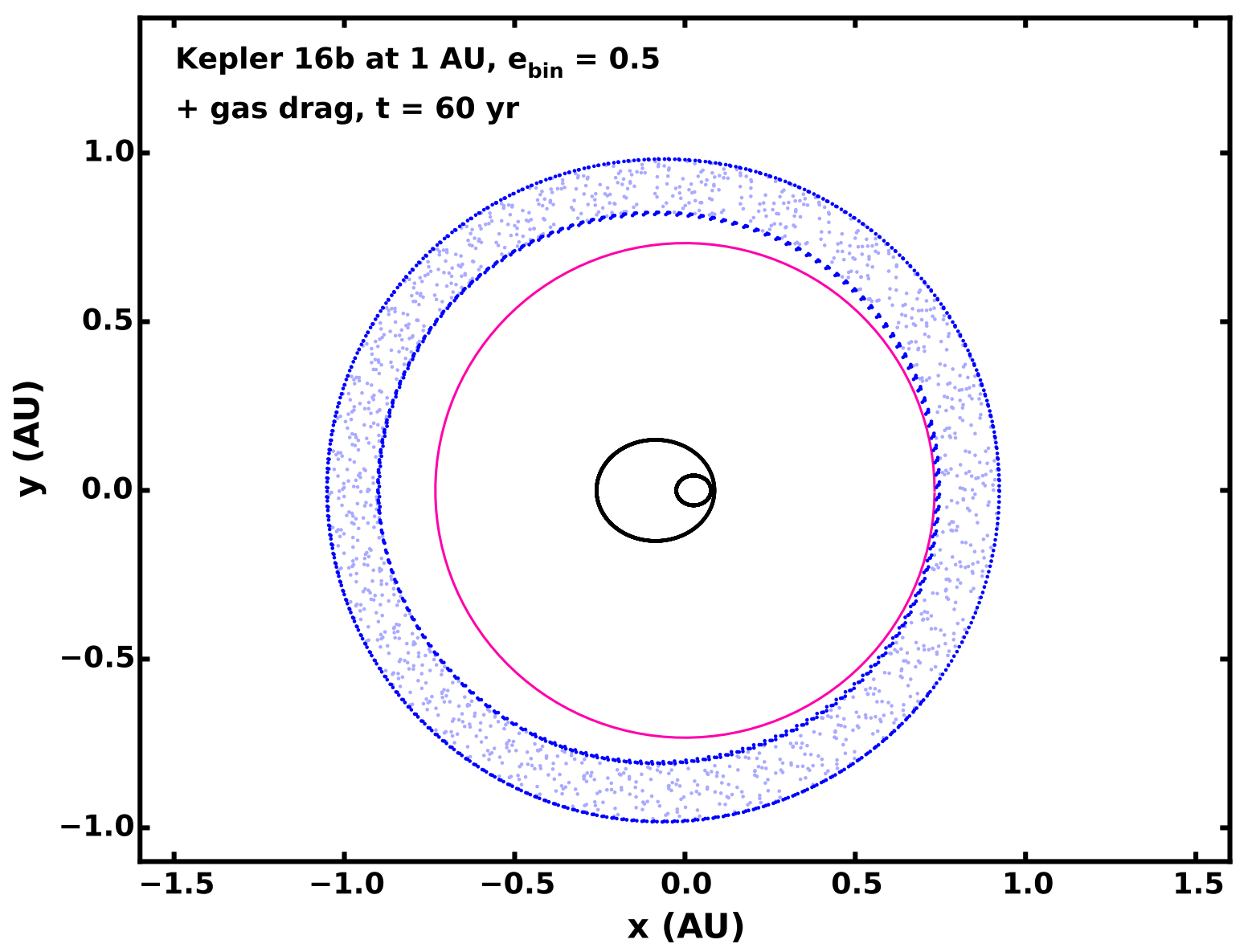

Fig. 5.- Inspiral from gas drag. A sub-Keplerian gas disk has fluid elements on most circular orbits with $\eta=0.001$ (Equation (40)). Embedded in it is a particle initially on a most circular orbit at $1 \mathrm{AU}$ (outer blue ring). The particle evolves, drifting inward as a result of a drag force proportional to its speed relative to the gas (light blue points; the local gas speed is calculated using the Lee-Peale-Leung analytical theory). The particle's final orbit (inner blue ring) remains apsidally aligned with the binary, a pair of stars like Kepler-16 except with $e_{\text {bin }}=0.5$ (inner black curves). A circular path (magenta curve) provides a reference. 


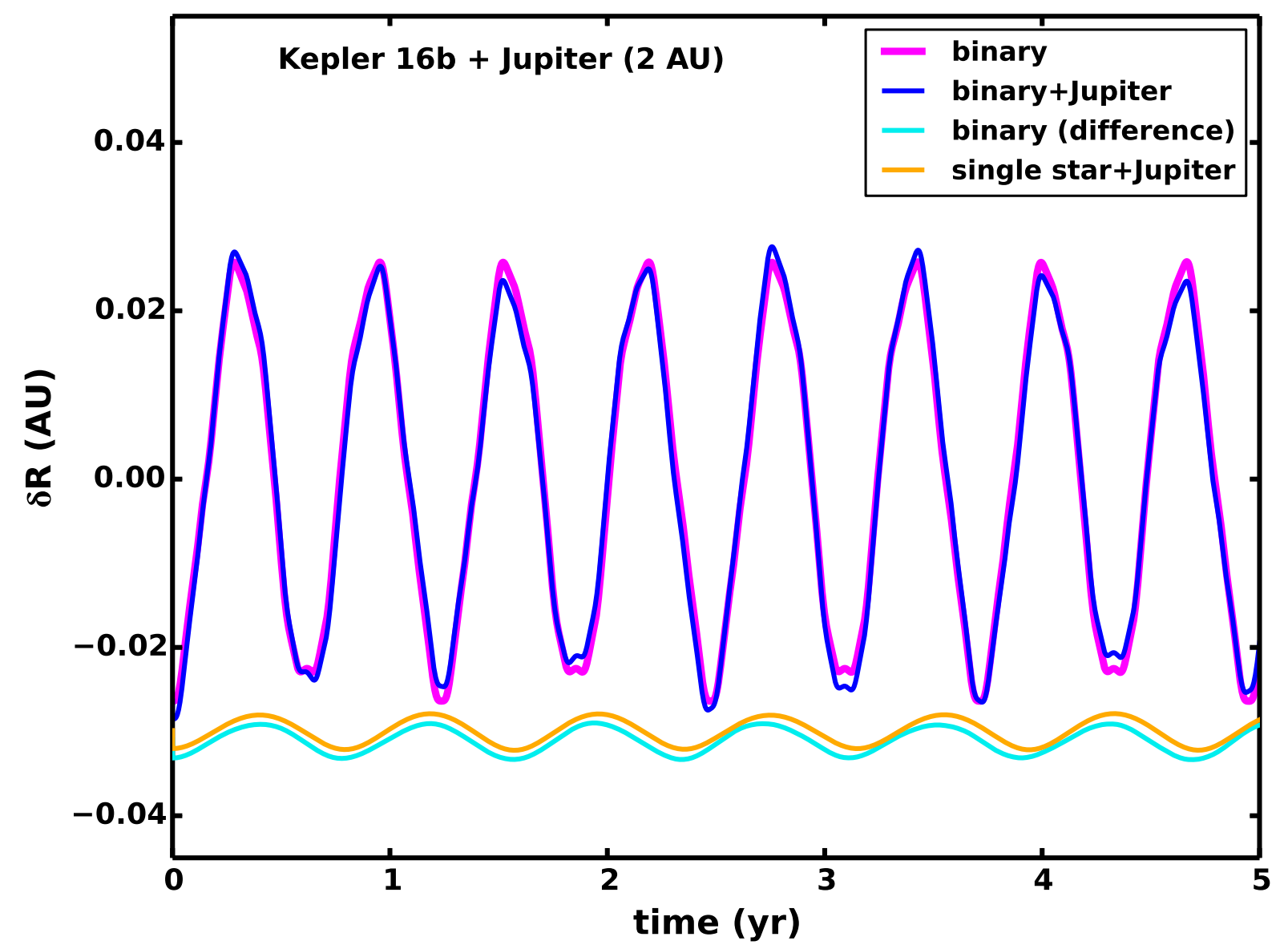

Fig. 6. - The effect of an external perturber on circumbinary orbits. The curves show most circular paths around Kepler-16 at the orbital distance of Kepler-16b, both in isolation (magenta curve) and with a Jupiter-mass planet orbiting with $e \approx 0$ at $2 \mathrm{AU}$ (blue curve). The difference between these two trajectories (cyan curve; offset for comparison) compares well with data from a satellite orbiting a single star with the mass of Kepler-16 and a Jupiter-mass companion (orange curve). These lower curves show that the most circular path provides a frame of reference for the action of external perturbations, just like a circularly orbiting guiding center in the circumstellar case. 


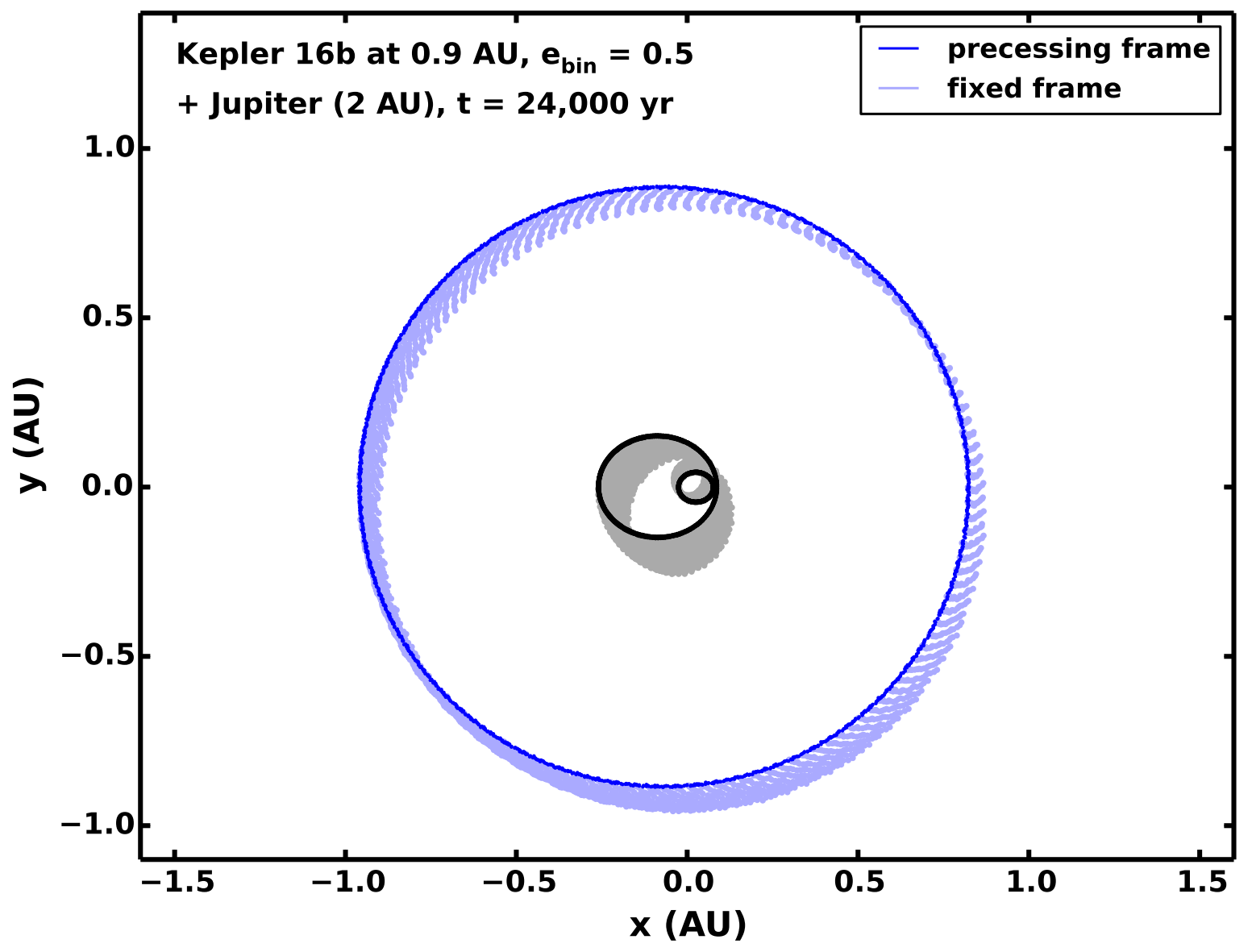

Fig. 7.- Binary precession and circumbinary orbits in a simulation. The outer curves show most circular paths ( $a=0.9 \mathrm{AU}$ ) around a binary like Kepler-16, set with eccentricity of 0.5 (inner curves) and with a Jupiter-mass planet orbiting with $e \approx 0$ at $2 \mathrm{AU}$. The gray and light blue-shaded curves show orbits in the inertial reference frame of the system's center of mass. The duration of the simulation is about a quarter of the precession period; orbits have precessed about $90^{\circ}$ (see Equation (56) ). The black and dark blue ellipses are the same orbits represented in a reference frame that precesses with the binary's periapse. The satellite's forced epicyclic motion evidently precesses at this rate. 


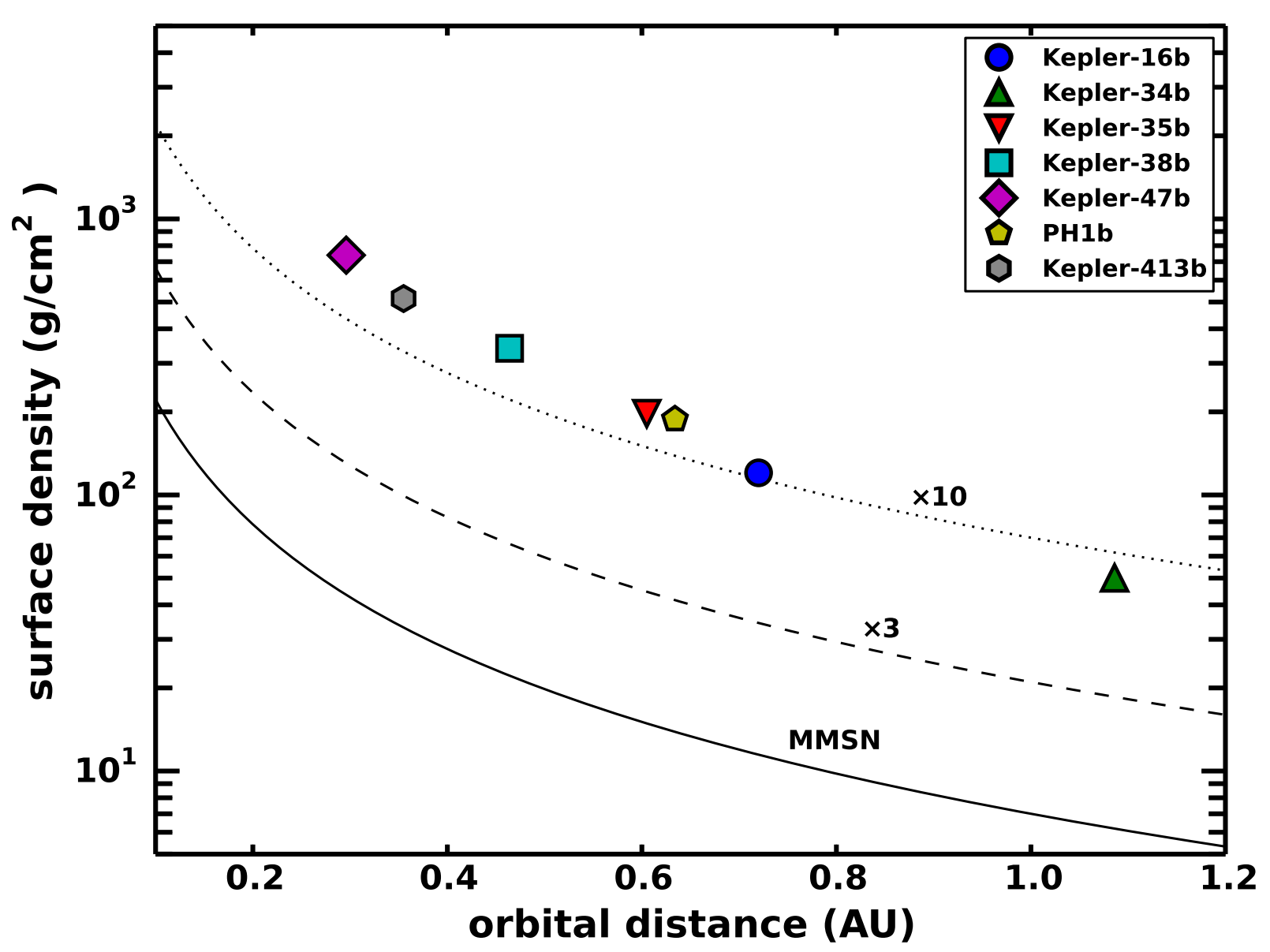

Fig. 8.- The minimum surface density to build the Kepler circumbinary planets. Each planet is shown at its orbital distance from the host binary. The value of the surface density $(\Sigma)$ comes from determining an annular width from which the planet could have accreted mass, based on its escape velocity when it had the mass of a $10 M_{\oplus}$, prior to its acquisition of a gas atmosphere (from Equation (65)). The solid line is the surface density of a Minimum Mass Solar Nebula $\left(\Sigma=7(a / 1 \mathrm{AU})^{-1.5} \mathrm{~g} / \mathrm{cm}^{2}\right)$, while the dashed and dotted lines correspond to disks with three and ten times that density (as labeled). Indications from simulations (e.g., Kenyon \& Bromley 2006) suggest that the intermediate-mass disk is a realistic starting condition for the Solar nebula. 

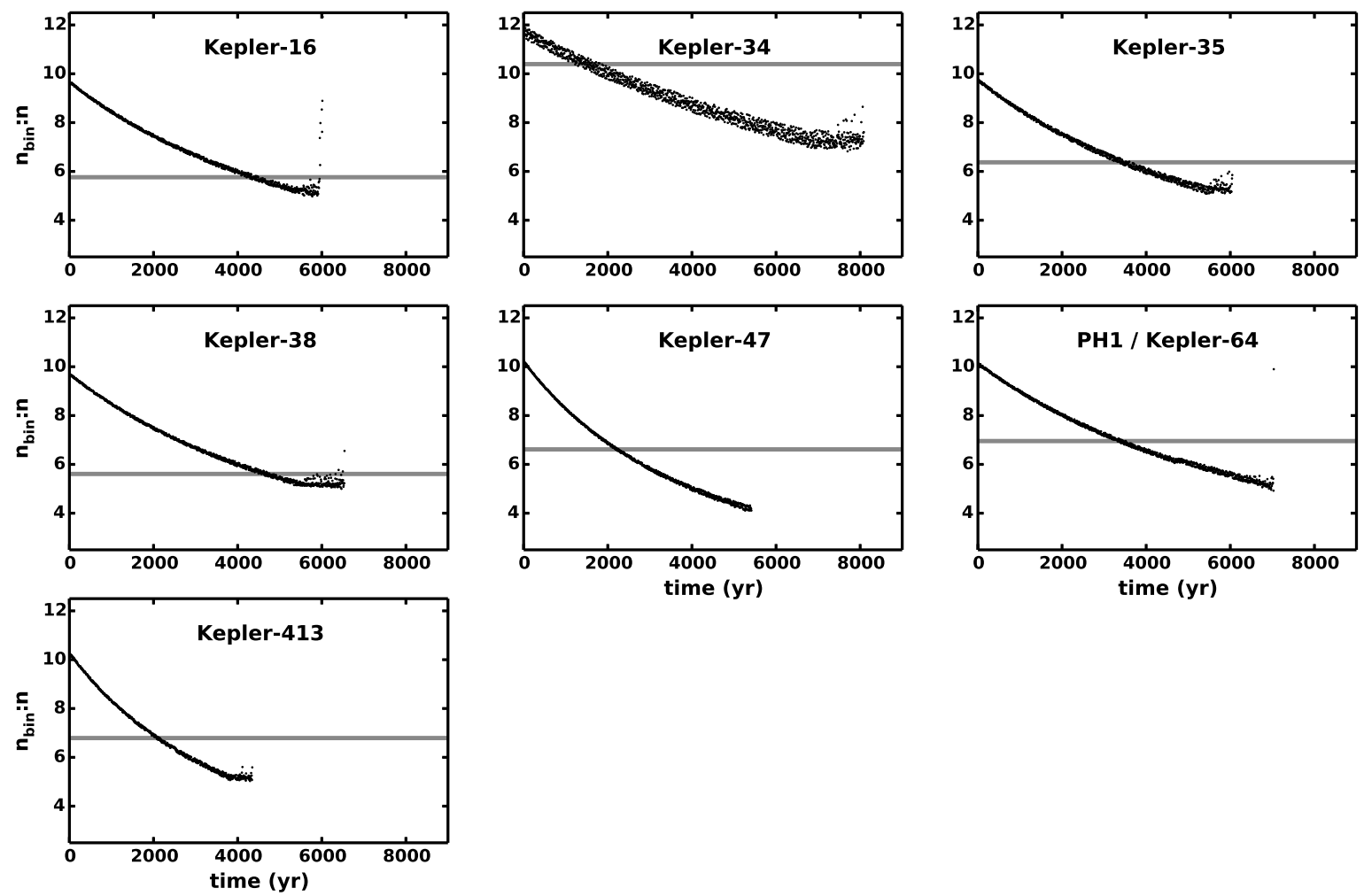

Fig. 9.- Stability of circumbinary orbits. Each panel shows the ratio of orbital frequencies between the central binary and the circumbinary planet. To mimic migration, we smoothly adjust the binary semimajor axis to cover the observed ratio of orbital frequencies (gray line). The end of each curve indicates where the orbit of the planet becomes unstable. For most planets, the 5:1 resonance is disruptive. Kepler-34b, whose binary host has high eccentricity $(e=0.5)$, goes unstable when it hits the 7:1 resonance. The planet around Kepler-47, with the lowest binary eccentricity of the group $(e=0.023)$, is stable down to the $4: 1$ resonance. Directly migrating the planet by artificially adjusting its semimajor axis gives similar results, except the planet around Kepler-34 remains bound. Here, we choose to gradually expand the binary's semimajor axis while preserving all other orbital elements. It is mathematically equivalent, and it is more straightforward to adjust the Keplerian orbit of the binary than the non-Keplerian orbit of the planet. 